\title{
Modelling the Diffusion and Operation of Anaerobic Digestions in Great Britain under Future Scenarios within the Scope of Water-Energy-Food Nexus
}

\begin{abstract}
The paper aims to understand the impacts of the spatial and temporal diffusion of Anaerobic Digestion (AD) on the Water Energy Food (WEF) nexus and to quantify the associated environmental, social and economic benefits. Contemporary tight carbon reduction targets urge the need to deploy renewable energy technologies however due to interdependencies across the WEF nexus, various technologies are beneficial for some but not all sectors. This paper quantifies the impacts of future possible AD technology diffusion choices on the environment, society and economy. This can aid decision makers to identify the potential consequences of various $A D$ alternatives within the next three decades. The study considers an integrated WEF nexus approach and accounts for the interdependencies within the nexus. This was done by developing an Agent-Based Model (ABM) and simulating the relations between the main players within the nexus, thus examining the upscaling of AD diffusion and its consequences for water consumption, energy production, transportation, landfill use, food waste processing and digestate generation. Three future WEF nexus scenarios, that reflect potential alternatives of society and technology in Great Britain up to 2050, were utilised by the $A B M$ implementation to test the sensitivity of $A D$ diffusion choices. These scenarios describe possible changes to lifestyle, governance, technologies, climate, and social structures. Accounting for the uncertainty associated with such future simulations, the Monte Carlo method was employed to estimate the potential variations in scenario outputs. Results suggest that decentralisation results in the largest carbon reduction, but can incur more costs. Centralisation consumes $35 \%$ more water but produces $37 \%$ more energy (biogas). The paper has visualised the scenario outputs graphically to highlight the consequences of neglecting the inter-relationships between environmental, social and economic aspects of AD.
\end{abstract}

Keywords: Anaerobic digestion; water-energy-food nexus; future scenarios; agent-based model; uncertainty. 
This section stresses the urgency of adopting renewable energy sources in Great Britain (GB) and promotes Anaerobic Digestion (AD) as a potential future technology. It then presents the significance of employing a holistic approach while considering the impacts on the WEF nexus when simulating the diffusion of an energy technology. The section identifies the gaps in the literature on modelling the performance and diffusion of $A D$ in GB using a holistic approach and recommends deploying agent-based modelling techniques.

\subsection{The future needs of energy in GB}

There is an urgent need to adopt renewable energy sources in order to meet the 2050 carbon reduction targets. The current GB contribution to reduce carbon emissions is much lower than some EU countries. For example, only around $7 \%$ of GB energy comes from renewables, which is much lower than other EU countries such as Sweden (48\%) and the EU average (16\%) (The Scottish Government, 2017). A significant power capacity from renewable sources, estimated to be between 25 and $70 \mathrm{GW}$, is required in GB to meet the 2050 targets (Li and Trutnevyte, 2017). The government plans to decarbonise energy by utilising renewable energy technologies (The UK Government, 2011) so there is a need to consider national effects of renewable technology alternatives. Future renewables can vary greatly in terms of their sources as there is uncertainty regarding the most viable technology. It is assumed that a portfolio of diverse renewable energy technologies will be required. Diffusing a well-known renewable technology that can utilise and feed into existing infrastructure is highly recommended for economical and other reasons (Eyre, et al., 2015). Energy produced from biogas via anaerobic digestion, which would utilise existing infrastructures for both production and distribution, can present a good potential for significant energy decarbonisation and it is being favoured, on a global level, as a sustainable renewable source (Sgroi, et al., 2015).

\subsection{Anaerobic digestion (AD)}

Anaerobic digestion produces biogas by converting most of the natural chemical energy, embedded in the biological waste, into energy through a low cost process (Batstone and Virdis, 2014) and can be implemented alongside other renewables to decarbonise energy in GB. Preliminary theoretical research has predicted a potential of producing 23.7 TWh/year from $A D$ biogas in $G B$, which represents over $22 \%$ of the electricity or $5 \%$ of the total annual national energy consumption (BEIS, 2018a). This prediction is based on annual methane production of 2344 million $\mathrm{m}^{3}$ (Scarlat et al., 2018) and a higher heating value of $55.5 \mathrm{MJ} / \mathrm{m}^{3}$ methane (Scarlat et al., 2018). Bio-matter (e.g. food waste) and biogas generated by the AD technology contain energy that can be stored. The time of use of these energy stores can be controlled to fit with demand, and so the technology is highly complementary to some variable and uncontrollable energy technologies, such as solar panels and wind power. An AD plant is scalable, where the operational principles can be implemented at different scales. This helps the spatial and temporal expansion of the AD technology depending on the energy demand variation, and can minimise the gap between energy supply and demand. Careful siting of $A D$ may lessen the dependence on the national gas grid connection and hence save costs and enhances the technology resilience. The end product of AD, i.e. biogas, is much less polluting than other fuels, e.g. the specific emission factor for typical electricity sources is 0.412 $\mathrm{kgCO}_{2} \mathrm{e} / \mathrm{kWh}$ which is more than double its biogas equivalent $\left(0.184 \mathrm{kgCO}_{2} \mathrm{e} / \mathrm{kWh}\right)$ (BEIS, 2018b). Previous authors, e.g. Evangelisti et al. (2014) assessed the environmental impacts 
of $A D$ in Greater London, UK, against other alternatives (incarnation with CHP and landfill with electricity production) to conclude that $\mathrm{AD}$ had the lowest emissions of $\mathrm{CO}_{2}$ and $\mathrm{SO}_{2}$.

\subsection{Holistic approach for innovation assessment}

Accounting for the interactions within the WEF nexus is crucial when considering the deployment of a novel technology. A number of researchers have stressed the necessity of employing holistic approaches when considering energy technologies, e.g. Kaddoura and El Khatib (2017). Others, such as Green et al. (2016), highlighted the importance of the nexus approach particularly in the private sectors since it can impact on human wellbeing. The latter authors identified the social and economic considerations as some of the key factors that can help companies manage the interdependencies within the WEF nexus. A holistic nexus approach has longstanding benefits and can optimise the use of resources and improve the economy, environment and health, while a single sector approach can be useful for the shortterm goals (Bazilian et al., 2011). The power of the nexus approach has helped researchers, e.g. Hatfield-Dodds et al. (2015), identify the real needs of mitigating the negative impacts of economic growth which were found to be mainly related to policymaking. Whilst a renewable energy technology, such as $A D$, might appear to independently reduce undesirable impacts on water, energy and food sectors, it is not clear how it affects the WEF nexus as a whole. This is due to the integrated nature of nexus sectors (direct feedback, as well as indirect feedback from the wider social-economic-environmental system in which it exists). Albrecht et al. (2018) have also stressed the importance of the WEF nexus approach and identified a need for a stronger consideration of the interactions between water, energy and food sectors, e.g. through the implementation of diverse methods from multiple disciplines.

The implementation of AD highlights the strong potential to achieve WEF nexus benefits, since its operation can result in significant water consumption to produce energy (biogas) while food waste can be a major $A D$ feedstock avoiding landfill. $A D$ was identified by previous authors as a prime solution for WEF nexus challenges (Haltas et al., 2017). Recent research, by Hoolohan et al. (2018), has emphasised the need for studying the deployment of AD within the WEF nexus to better understand the interactions between various fields. Benefits arising from AD implementation, within the WEF nexus include the contribution to waste disposal reduction which can consequently reduce carbon emissions of conventional landfill incarnation processes (National Grid, 2016). AD digestate, the residue of food after bio-gas production, can be used for agricultural purposes, by providing crops with the readily available nitrogen and substitute synthetic fertiliser, which would therefore cut costs and carbon emissions associated with fertiliser production (WRAP, 2012). Other nutrients available in AD digestate, mainly phosphate and potash, are valuable for maintaining soil fertility and water retention, which can reduce the agricultural water demands (WRAP, 2012).

In many studies, modelling the viability of an innovative renewable energy technology has been approached by predicting its environmental, e.g. Whiting and Azapagic (2014), and its economic, e.g. Lauer et al. (2018), impacts in isolation from the potential consequences that may incur as a result of the technology implementation. This does not sufficiently consider the whole system or nexus effects. For example, implementing AD on centralised or decentralised national levels is interlinked with the water and energy consumed for operating the plant, people's diet, food waste transportation, available land and the public acceptability of the new technology. Neglecting the interconnectivity between systems may undermine the potential 
implications on a holistic level. Other studies used more holistic approaches to evaluate the impacts of utilising various municipal solid waste technologies, including $A D$, on energy, economy and environment, e.g. Tan et al. (2015). However, the latter authors focused on a specific region of implementation in Malaysia and have not accounted for the consequent implications on the WEF nexus. The future scenarios of Tan et al. (2015) were limited to two categories; isolated and integrated and there was no comprehensive representation of the potential future societal, technical and environmental changes. Fernández-González et al. (2017) analysed the implications of implementing several waste to energy technologies, including $A D$, in terms of economic, social and environmental values in a specific Spanish geographical region but have not considered the interdependencies within the WEF nexus. Pacetti et al. (2015) assessed the impacts of biogas production from AD on the water and the environment by using an innovative method that combined Water Footprint and Life Cycle Analysis. Their study focused on three regions in Italy to compare various crops and treatment processes used for AD. However, Pacetti et al. (2015) have not accounted for the social and economic impacts of the likely future variations in lifestyles and economies that would influence the potential alternatives of $A D$ performance, food usage and energy production and consumption. The aforementioned studies analyse and compare alternatives of implementing potential waste-to-energy technologies while not necessarily accounting for future implications caused by the likely changes in society, lifestyles, clean energy availability and the economic structures within the WEF nexus.

Other studies have focused on improving the assessment of integrated waste management systems considering a holistic approach. For example, Rigamonti et al. (2016) defined three comprehensive indicators that enable the assessment of the environmental and economic sustainability of integrated waste management systems. Two of the indicators assess the environmental impacts by accounting for the ratios of energy and materials recovery and recycle respectively while the third indicator evaluates the costs of the system. The three indicators, defined by Rigamonti et al. (2016), can be used by technical and non-technical stakeholders to compare the performance of various integrated waste management systems. However, the latter authors have excluded any indicators that can be utilised to assess the social dimensions. Whilst other researchers, e.g. Bazilian et al. (2011), developed modelling conceptual frameworks that are specifically designed to quantitively address the WEF nexus, there is a gap in the literature for methods that quantify social, economic and environmental impacts, within the WEF nexus, of a potentially viable technology. Overall, there is a gap in holistic consideration of environmental, social and economic impacts, within the WEF nexus, when quantifying the performance and diffusion of $A D$.

\subsection{Agent-Based Modelling (ABM)}

Despite the advantages of the nexus approach, it has a number of challenges, mainly regarding the understanding and modelling of the complex relations between sectors. Modelling such relations can be achieved using various methods, e.g. agent-based, empirical, system dynamics, economic theory, topology-based and flow-based techniques (Ouyang, 2014).

Some of these, e.g. the empirical methods, cannot capture the impacts of system failure, while others, e.g. the economic theory methods, cannot function at a component level (Momeni et 
al., 2018) which can be problematic when simulating AD performance in this study. The flowbased method can provide an accurate model framework when implemented with high temporal resolution, but this may be computationally expensive (Momeni et al., 2018).

The selection of an appropriate modelling method should be based on the application. System Dynamics or ABM techniques can be used in this study as they can quantify the cascading effects (Iturriza et al., 2018). The System Dynamics method has a reputation of being reliable in analysing complex interdependent systems (Zio and Sansavini, 2011), but it requires calibration (Momeni et al., 2018), which may prove challenging to achieve in this study due to the lack of data.

Various players exist in nexus systems, with their relevant objectives, priorities and boundaries that influence their actions, while interacting with each other within an evolving environment. $A B M$ is a technique used to simulate the interactions between different organisations (agents), while accounting for each agent's contribution. Quantifying various impacts using 'what if' analysis is supported by ABM, which can detect unexpected outcomes and therefore can enhance the quality of decision making guidance (Kaegi, et al., 2009). Modular structure and scalability are significant advantages of using ABM to model an innovation within the nexus at the desired scale and level of complexity. The ABM is also capable of simulating complex systems (Cardellini et al., 2007) with various subsystems at different abstraction levels (Casalicchio et al., 2007). Furthermore, ABM was found, by previous authors such as Rinaldi (2004), to be versatile and can be integrated with other modelling techniques to enrich its capabilities. Accounting for future uncertainties can be accomplished through ABM by considering a range of input parameter values and employing the Monte Carlo method to produce a range of potential outputs. Whilst the main disadvantage of the ABM approach is the demand for large datasets, this problem is common with most methods (Momeni et al., 2018), and its consequences can be mitigated by making realistic assumptions, when needed, based on expert knowledge.

Few researchers have addressed the diffusion of an $A D$ related technology using $A B M$. For example, Sorda et al. (2013) utilised ABM to investigate the impacts of new financial subsidy changes on the diffusion of combined heat and power engines (CHP) that are fuelled by agriculture waste in two German states. Other authors, such as Faber et al. (2010), have developed ABMs to simulate the cost differences between micro-CHP and condensing boilers in the Netherlands. The latter study was based on demand, and therefore used housing types to represent the consumer agents. The consumers decide the uptake of the technology based on their need, awareness of the technology output and the associated costs. The study by Faber et al. (2010) was useful for estimating the required purchase subsidy and conditions for micro-CHP diffusion but did not account for social and environmental impacts within the WEF nexus. The diffusion of energy fuels was also modelled by Tran (2012), while considering the social impacts. In his ABM, Tran (2012) accounted for the individual and network impacts on energy diffusion. He considered direct and indirect social influences that arise from close neighbours and the general public, respectively. Tran's study focused on consumer decision making and how it can influence the acceleration of energy diffusion, but did not account for the potential future variations in lifestyles, social structures and strategic changes. 
Although ABM is considered a promising tool for assessing the resilience of energy system implementations (Toth et al., 2016), the literature review has indicated a gap in utilising ABM for quantifying the potential $A D$ benefits across $G B$. Hence, there is a need for utilising $A B M$ capabilities, i.e. simulating interconnections and the modular and scalability features, to quantitatively forecast the impact of $A D$ diffusion on the WEF nexus at a national level. The implementation of ABM needs to incorporate the future scenarios around environmental, social and economic aspects to account for the uncertainties associated with the WEF nexus.

\subsection{Future scenarios within the WEF nexus}

The future holds a great deal of uncertainty particularly in the WEF area. Therefore, accounting for potential changes in lifestyles, social and economic structures and strategic changes is important when simulating the diffusion and performance of a new renewable energy technology. This led Hoolohan et al. (2019) to create three non-probabilistic scenarios using qualitative methods to reflect the potential societal, technical and environmental changes over the next three decades for the WEF nexus. Each scenario describes changes in climate impacts (i.e. the changes in climate to be experienced in 2050); climate action (i.e. the extent of action taken to mitigate the climate change); and governance (the arrangements, goals and processes that affect change in the society). These three dimensions were used to frame assumptions regarding the nature of social, technological and climatic changes experienced by 2050. Narrative detail was developed based on existing scenario studies of the future of water, energy and/or food systems within GB.

Hoolohan et al., 2019 conducted a workshop to substantiate the scenario narratives and to specify the possible implications for nexus-innovations, including AD. Thirty-one participants were assembled, reflecting a range of experience, responsibility and vision. Participants included individuals actively involved in anaerobic digestion, or two other nexus-innovations, along with individuals from nongovernmental and charitable organisations, the private sector and consultants, all with specialisations relevant to the WEF nexus. Facilitated workshop discussions enabled participants to interrogate the assumptions made in the scenarios, and to consider the nature and functioning of $A D$ in conditions that are radically different from today. Table 1 describes the key characteristics of the scenario narratives along with the possible implications for AD, identified by participants within the workshop. This method enables complex systemic change to be described, offering rich descriptive detail to couple with quantitative assumptions to inform the ABM. 
Table 1: Key characteristics of the initial scenario narratives, and the specified possible implications arising from workshop discussions. Adapted from Hoolohan, et al. (2019).

\begin{tabular}{|c|c|c|}
\hline Scenario & Generic characteristics & Specified implications for AD \\
\hline $\begin{array}{l}\text { Share and } \\
\text { Connect }\end{array}$ & $\begin{array}{l}\text { A decentralised society with high levels of } \\
\text { digitisation, high levels of connection between } \\
\text { producers, consumers and the environment, } \\
\text { and low levels of material consumption. } \\
\text { Energy and water demand are low and } \\
\text { emissions are in-line with the Paris } \\
\text { Agreement's ambition of limiting global } \\
\text { average temperature rise to } 2^{\circ} \mathrm{C} \text {. This is due } \\
\text { to a combination of lifestyle change and } \\
\text { sophisticated tools for remote control. Food } \\
\text { production, retail and catering have become } \\
\text { more diverse with a greater number of small- } \\
\text { to medium-size enterprises. Renewable } \\
\text { energy and waterless technologies are } \\
\text { pervasive and novel agricultural systems } \\
\text { allow wider participation in the provision of } \\
\text { water, energy and food. }\end{array}$ & $\begin{array}{l}\text { - AD becomes a flexible technology for } \\
\text { nutrient management, organic waste } \\
\text { management and in some areas, energy } \\
\text { generation, though energy demand is low. } \\
\text { - Local stakeholders buy into AD as it } \\
\text { becomes a rich area for community } \\
\text { investment. Commercial plants open } \\
\text { community share schemes. } \\
\text { - In other cases, small specialised plants } \\
\text { provide communities with bespoke } \\
\text { resource management solutions. Digestate, } \\
\text { biogas and biofuels are used for local } \\
\text { needs. } \\
\text { - Decisions on planning, siting and dispute } \\
\text { resolution take place in community interest } \\
\text { groups. }\end{array}$ \\
\hline $\begin{array}{l}\text { Create } \\
\text { and Cope }\end{array}$ & $\begin{array}{l}\text { A society experiencing climate impacts } \\
\text { sooner and more extreme than anticipated, } \\
\text { with greenhouse gas emissions remaining in } \\
\text { line with the current GB legal obligations ( } 80 \% \\
\text { cut from } 1990 \text { by 2050). Vibrant } \\
\text { entrepreneurial activity is sparked by the } \\
\text { declining function of centralised systems of } \\
\text { provision. Climate change has noticeable } \\
\text { biophysical effects, prompting rapid and } \\
\text { transformative changes to everyday life. The } \\
\text { scarcity of resources drives the circular } \\
\text { economy into practice, and waste is a } \\
\text { precious source of materials. The key } \\
\text { characteristic of this scenario is the } \\
\text { differentiated impacts and adaptations } \\
\text { experienced across GB. }\end{array}$ & $\begin{array}{l}\text { - } A D \text { innovation and deployment vary } \\
\text { according to local social and climatic } \\
\text { conditions. For example, with high fuel } \\
\text { costs } A D \text { is a valuable source of } \\
\text { biomaterials in agricultural areas, while } \\
\text { households and businesses use } A D \text { to } \\
\text { produce growing material, energy and other } \\
\text { outputs, depending on their needs. } \\
\text { - AD sees rapid innovation to enhance non- } \\
\text { energy outputs. } \\
\text { - Small scale, privately owned AD is common } \\
\text { in both rural and urban areas. and } \\
\text { largescale } A D \text { is also used to process } \\
\text { inedible wastes to produce biomaterials, } \\
\text { fuel, chemicals, digestate and gas. }\end{array}$ \\
\hline $\begin{array}{l}\text { Big and } \\
\text { Smart }\end{array}$ & $\begin{array}{l}\text { A highly centralised digital society where big } \\
\text { infrastructure supplies basic needs, heavily } \\
\text { regulated for transparency and efficiency. } \\
\text { Fossil energy supply systems have declined } \\
\text { following GB commitments to the Paris } \\
\text { Agreement (though are misaligned with the } \\
2^{\circ} \mathrm{C} \text { goal), due to extensive investments in } \\
\text { large-scale renewables, accompanied by } \\
\text { some nuclear and bioenergy. Climate- } \\
\text { controlled food production is now necessary, } \\
\text { which means big agricultural firms have the } \\
\text { majority of the market. This is a big data } \\
\text { driven society allowing demand driven supply } \\
\text { chain efficiencies. }\end{array}$ & $\begin{array}{l}\text { - AD is the route for processing inedible } \\
\text { organic wastes with improved digestate } \\
\text { quality. } \\
\text { - Large indoor farms have AD built in and bio- } \\
\text { refineries produce energy, biomaterials, } \\
\text { chemicals, and pharmaceuticals from } \\
\text { hybridised feedstocks. } \\
\text { - Public services (hospitals, universities etc. } \\
\text { have smaller digesters on-site). } \\
\text { - The delivery of digestate to soils is } \\
\text { optimised for example using regulatory } \\
\text { mechanisms and automated technologies. }\end{array}$ \\
\hline
\end{tabular}




\subsection{Summary, novelty, aim and objectives}

Sections 1.1 and 1.2 have shown the importance of adopting scalable renewable energy technologies in GB and presented $A D$ as a potential technology, due to its various advantages including the utilisation of existing infrastructures. It is clear from Section 1.3 that a holistic approach is required when investigating the deployment of a renewable energy technology to address the various impacts within the WEF nexus. The literature review has indicated a gap in quantifying the social, economic and environmental impacts of AD within the WEF nexus as explained in Section 1.3.

Modelling of holistic systems demands approaches that are sensitive to interdependencies and dynamics, and Section 1.4 has shown the value of ABM to address such traits of complex systems. Despite the capabilities of ABM to simulate interconnections within the WEF nexus, it was not deployed previously to model the performance and diffusion of $A D$ in GB (Section 1.4). Thus, there is a gap in the simulation of $A D$ diffusion and operation at a national scale (across GB) while considering a holistic approach.

The novelty of this study lies in the simulation of $A D$ diffusion and operation at a national scale (across GB) using a bottom-up agent based modelling approach, while accounting for the forecasted social, economic and environmental changes and quantifying some of the relevant effects on the WEF nexus. The paper deploys ABM and uses future scenarios that reflect various potential alternatives of societies, lifestyles, economic structures and types of energy systems. The scenarios considered in this paper were developed by Hoolohan et al. (2019), as part of project (Stepping UP) and are used for the first time to simulate the impact of $A D$ diffusion on the WEF nexus. The utilised approach addresses all four key features (i.e. innovation, social and political context, collaboration and implementation) identified by Albrecht et al. (2018) for developing a promising tool to guide decision makers and future research.

The aim of the paper is to understand the impacts of the spatial and temporal diffusion of $A D$ on the WEF nexus and to quantify the associated environmental, social and economic benefits. To do this, there is a need for developing and deploying an $A B M$ to simulate the diffusion of $A D$ across $G B$ using various future hybrid energy system scenarios. The $A B M$ will also be utilised to quantify the environmental $\left(\mathrm{CO}_{2}\right.$ emissions), social (acceptability of $A D$ plants and landfills production) and economic (costs and costs avoided) impacts of $A D$ implementation on the WEF nexus.

Section 2 provides a detailed methodology and research design for data management and modelling of $A D$ diffusion at a national scale. Section 3 presents the results of the modelling, whilst Section 4 contains a discussion of the findings. Section 5 concludes the paper and outlines limitations. 


\section{Methodology}

This section briefly explains the procedure followed in this research, the future scenarios that will influence $A D$ implementation and describes how an $A B M$ was developed to simulate the diffusion and operation of AD plants in GB between 2017 and 2050.

\subsection{Research Design}

Anaerobic digestion is an innovative technology expected to have considerable influence on the way future energy is produced (see Section 1.2). The impact on the WEF nexus due to diffusion of $A D$ across GB depends on future lifestyles and associated social structures. The strategy used in this paper is to quantify the impact of $A D$ diffusion of alternative lifestyles by assessing how they affect a baseline future which consists of 'more of the same' as today. Future scenarios, developed by Hoolohan, et al. (2019), that embrace WEF considerations and expand on relevant lifestyles across the next three decades, were utilised in this paper. These scenarios consider how the society might be organised (centralised versus decentralised) and how societal lifestyles would change. The qualitative narratives of the scenarios were interpreted to quantify the changes to baseline parameters. The values were supplied to an $A B M$ developed for this purpose so the ABM could assess the range of environmental, social and economic impacts of the trade-offs at the WEF nexus. Figure 1 presents the research problem in broader terms and the scope if this study.

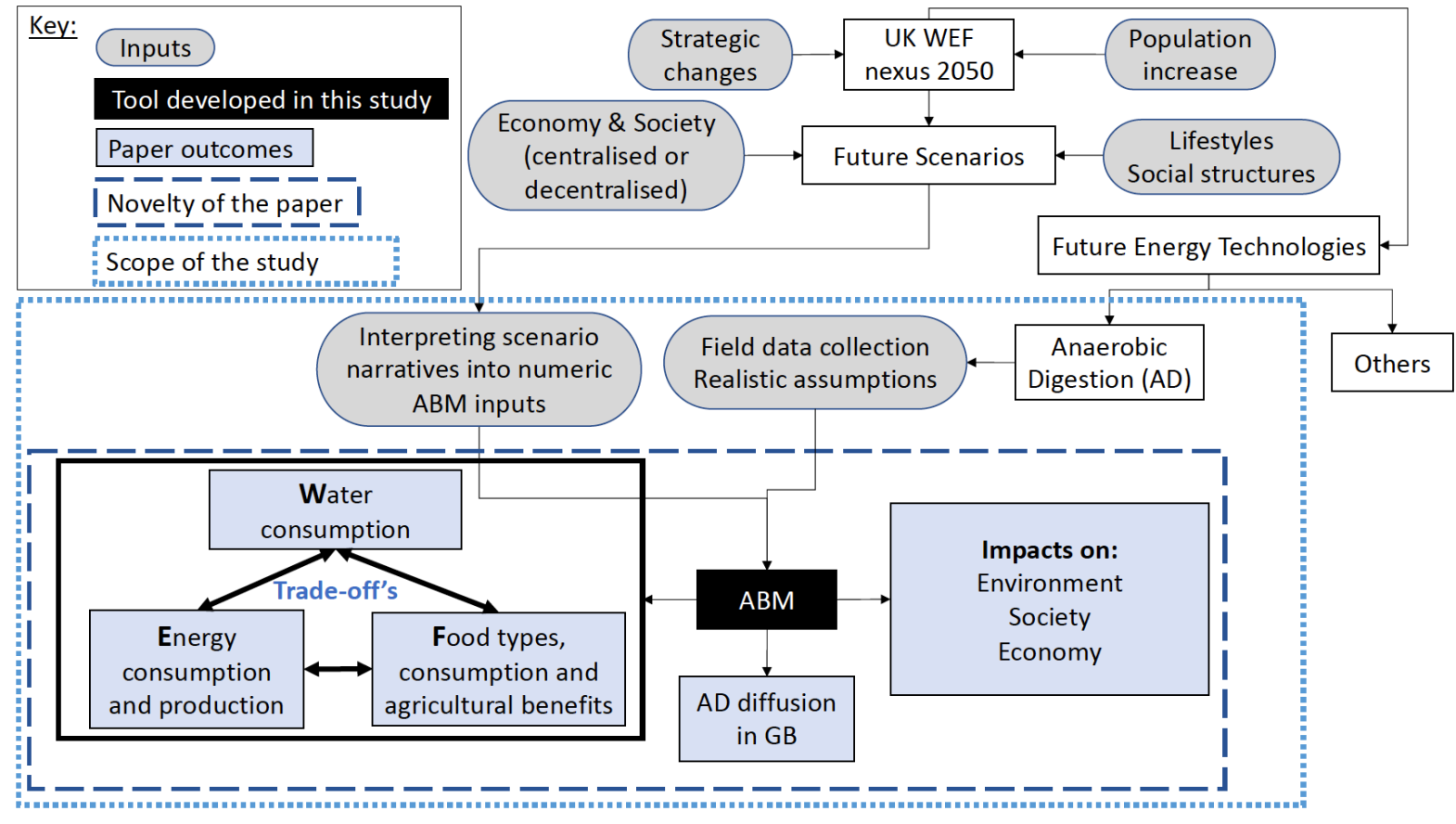

Figure 1: Scope of the research problem.

\subsection{Interpreting scenario descriptions into $A B M$ numeric input parameters}

The scenario narratives, described in Section 1.5, were interpreted into numeric values to be used as ABM input parameters. The Baseline values reflect recent (year 2017) conditions, which were retrieved from the literature, measured data and realistic assumptions based on expert knowledge. The future of the Baseline excludes any radical technological and lifestyle changes, but accounts for conventional anticipated variations, e.g. in population growth and operational costs. Most of the Baseline inputs had value ranges that accounted for the 
parameter uncertainty and future variations. The change within each range was assumed to be uniform, which is a fair assumption due to the lack of real accurate data to estimate the actual values in most cases. The input parameters for future scenarios were estimated based on the future changes described by the scenarios, in reference to the Baseline. Table 2 lists the $A B M$ input parameters and their estimated values.

\subsection{Excluding Create \& Cope in the ABM}

Create \& Cope is a deeply complex scenario; not only are there many uncertainties, but the dominant logic of Create \& Cope is that there is also no overarching trend for how social, technological and climatic changes play out across GB, and subsequently no homogenous pattern for $A D$ development across GB. The pace and nature of innovation implementation and diffusion, in this scenario, differs spatially across GB due to varying economic investments, experiences of climate change and opportunities for industrial adaptation. Climate change is experienced differently in different regions, which has implications on the routines of people and organisations. For example, the immediacy of water scarcity in the Southeast could lead to different models of water service provision, e.g. water-less laundrettes. Consistent with this scenario, the pattern of $A D$ development and diffusion is suggested to vary, with plants of different scales being used for specialised applications. This affects both the direction of $A D$ innovation, e.g. $A D$ would principally act as an energy technology in some areas, while in others its potential to offer digestate and biochemicals would be favoured; and the scale on which it is deployed, the location and the actors involved. This variability is pronounced in different regions, but also across them with a high level of diversity and dynamism characteristic of Create \& Cope.

Since the ABM designed for this study simulates a single trajectories across $G B$, and not different trajectories of innovation diffusion in different geographical areas across $\mathrm{GB}$, the Create \& Cope scenario is not addressed in this paper. 
Table 2: Input parameters for the ABM

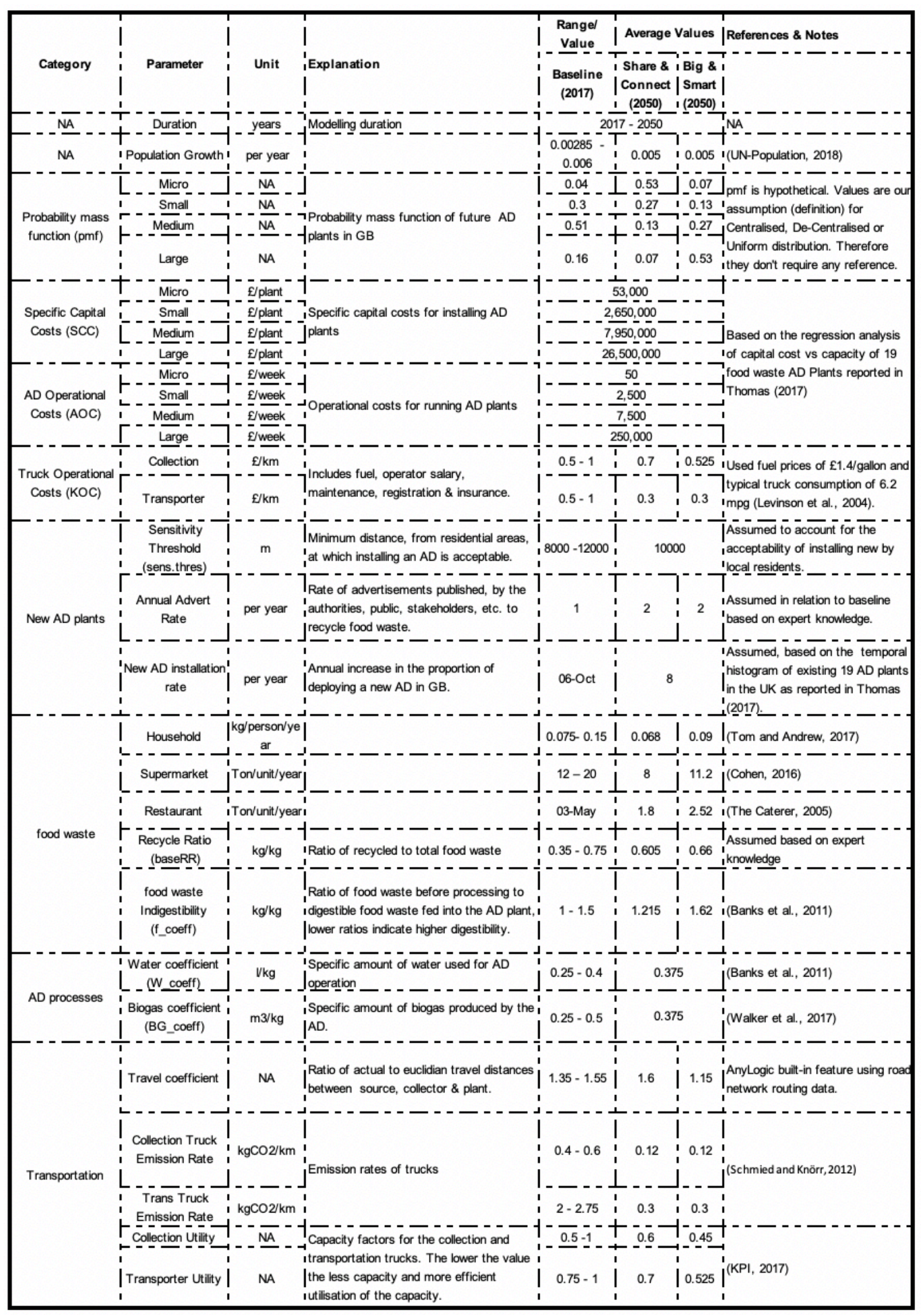




\subsection{Agent-Based Model: Overview, Design concepts, and Details (ODD) ${ }^{*}$}

The description of the ABM is summarised in this section following the ODD protocol (Grimm et al., 2006 and 2010). ODD is a well-known protocol created to standardise the methodology of developing an ABM. The modelling platform for the ABM is the AnyLogic 8.2.3 Researcher Version. AnyLogic is a multi-method simulation software that is designed to support the development of agent based, discrete event and system dynamic models (Grigoryev, 2015). Many built-in graphical tools and functions, embedded GIS and database support significantly simplify model development with minimal coding requirements.

\subsubsection{Purpose}

Simulate the diffusion of $A D$ and quantify the environmental $\left(\mathrm{CO}_{2}\right.$ emissions), social (acceptability of $A D$ plants and landfill production) and economic (costs and costs avoided) impacts of $A D$ diffusion across GB.

\subsubsection{Agents:}

1. Main: Manages the input parameters and triggers events.

2. DummyCollector: Represents the feasibility investigation for new food waste collectors and assigns the relevant sources.

3. DummyPlant: Determines the location of a new Plant.

4. Collector: The food waste collector.

5. GridCell: The study area is mapped with $50 \mathrm{~km}$ resolution GridCells to perform an efficient search for the best new collection area locations, based on the available generation potential. There are 196 GridCells covering GB.

6. Plant: AD Plants.

7. Scenario: Holds the values of model input parameters for each scenario.

8. Source: Represents the sources that are assigned to a collector, includes households, restaurants and supermarkets.

9. SourceArea: Represents the food waste collection areas within $\mathrm{GB}$, set as middle layer super output area (MSOA).

10. SubGridCell: The study area is further mapped with $5 \mathrm{~km}$ resolution SubGridCells. The DummyPlant searches the best location for the new plant among the SubGridCells near the assigned collector. There are 13110 SubGridCells covering GB.

\subsubsection{Process overview}

The diffusion and operation of AD plants are simulated in parallel. The model starts with the existing SourceArea, Collector and Plant agents. In the existing operations; the AD feedstock, i.e. food waste, is generated at the SourceAreas, collected by the collectors and transported to the plants for processing, which produces biogas and digestate. The DummyCollector initiates feasibility search for new collection areas according to its behaviour rules (see Section 2.4.4). If the new search results in success, a new source is created within the SourceArea and the DummyPlant starts a search for the location of the new AD plant. This search populates the Plant agent with a new AD. The newly populated Source and Plant agents are added to the existing operations and these diffusion and operation processes continue until the end of the simulation which can vary from one day to 33 years. Figure 2 shows schematically the main simulated processes and interconnections described above.

\footnotetext{
* Requests for data and code should be sent to researchdata@cranfield.ac.uk
} 


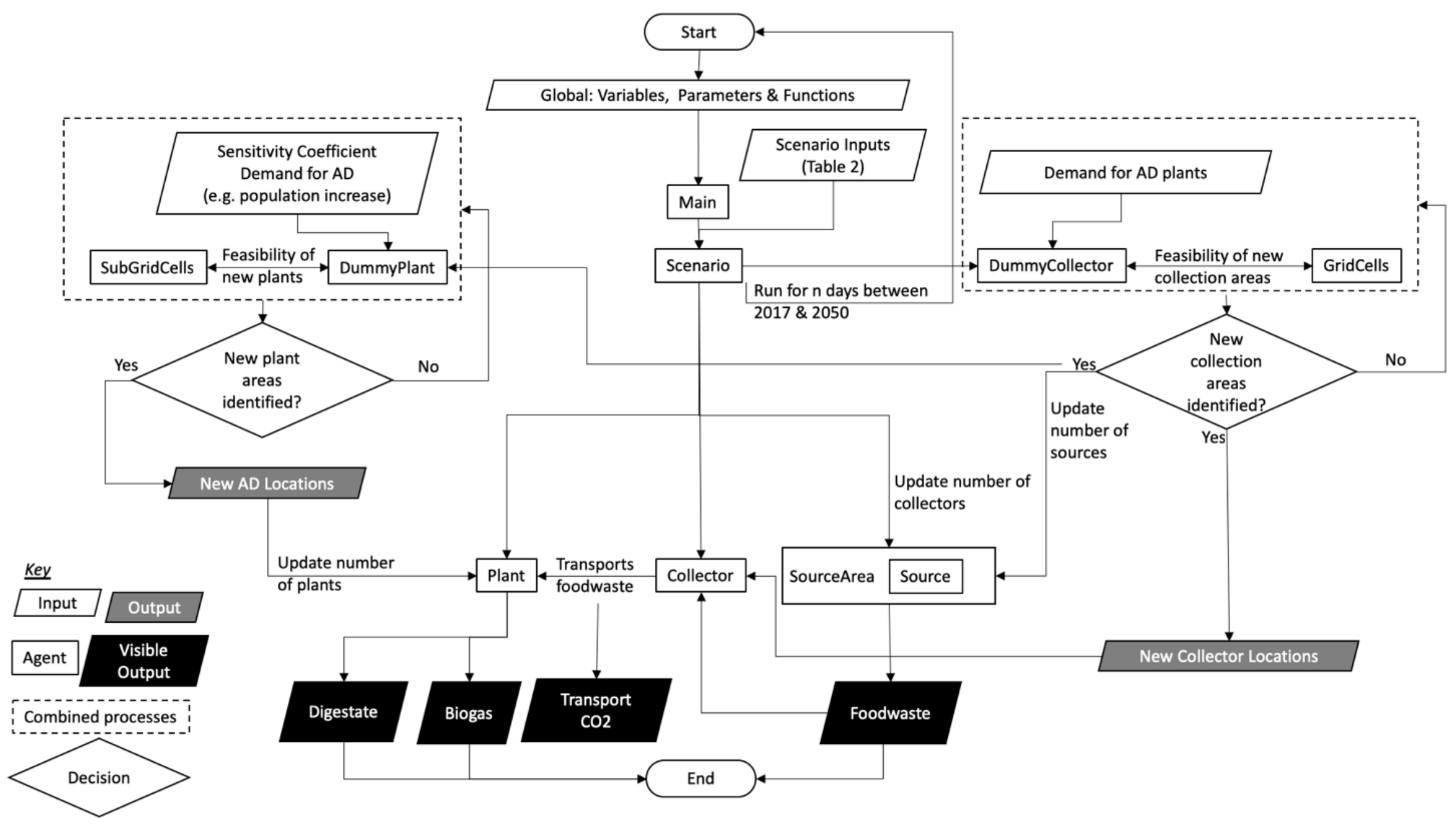

Figure 2: Flowchart of the ABM showing the main simulated processes and interconnections. 


\subsubsection{Behaviour rules for each agent.}

1. Main:

- No spatial location.

- Selects the class (micro, small, medium or large) and capacity (tonne/day) of the next Plant based on the given probability distribution.

- Triggers a feasibility search for a new operation (Plant/Collection) at a given frequency (times per year).

- Triggers a 'shutdown' search among the existing plants as set by the probability mass function (Table 2). A plant 'shutdown' results in deactivation of the relevant collectors and food waste Source(s) assigned to the plant.

- Records various model parameters and variables into the output file.

2. DummyCollector:

- Spatial location is dynamic

- A single agent is populated at the beginning of the simulation.

- When a new feasibility search is triggered by the Main agent, DummyCollector searches for the GridCell with the highest potential for a new collection point.

- If the feasibility search is successful, i.e. a potential location is identified, the following actions occur;

- DummyCollector locates itself at the centre of the corresponding GridCell.

- DummyCollector assigns sources from the nearby SourceAreas with available food waste until the capacity of the proposed plant is met.

- DummyCollector locates itself at the centre of the assigned sources and a new collector is installed (Collector agent is populated) at the identified location.

- A command is triggered to search for a new plant location.

- If the feasibility search is unsuccessful, i.e. no potential for a new plant, then this search event is skipped and DummyCollector waits until the next feasibility search.

3. DummyPlant:

- Spatial location is dynamic.

- Populated with a single plant at the beginning of the simulation.

- When a new Plant location search is triggered by the DummyCollector, DummyPlant searches for the SubGridCell closest to the assigned collector and with a sensitivity index lower than the given threshold.

- A new Plant is installed (Plant agent is populated) at the identified SubGridCell.

4. Collector:

- Spatial location is static.

- Initial population is calculated based on the existing number of $A D$ plants and SourceAreas.

- Dynamically generates new collectors if the DummyCollector feasibility search is successful.

- Collects from the assigned active sources weekly.

- Transports the collected food waste to the assigned plant.

- Calculates the collection and transportation costs and carbon emissions.

5. GridCell:

- Spatial location is static.

- Initially populated with a fixed number from an existing database. 
- Potential of food waste generation (total, available and adopted) of a GridCell is calculated and updated daily.

6. Plant:

- Spatial location is static.

- Initial population (existing AD plants) is loaded from a database retrieved from the literature.

- New plants are dynamically generated if a feasibility search by the DummyCollector is successful.

- An active plant processes the food waste and converts it to biogas and digestate. Water is consumed in the process.

- A Plant is deactivated if the Main agent triggers a shutdown.

7. Scenario:

- No spatial location.

- Initially populated from an existing database.

- Updates the values of model input parameters gradually every year from initial values to final scenario values.

8. Source:

- Spatial location is static.

- Food waste is assumed to be the only AD feedstock due to the lack of data from other sources

- Dynamically generated population, initially empty.

- A source is populated in active status within a SourceArea.

- If the assigned Collector/Plant is 'shutdown', the source becomes inactive.

- Active source generates food waste at a generation rate.

- Active source recycles food waste at a recycling rate.

- Recycle ratio fluctuates over time depending on various (implicit) factors.

- Non-recycled (dumped) food waste goes to the landfill.

9. SourceArea:

- Spatial location is static.

- Initially populated with a number from an existing database.

- Number of households, restaurants and supermarkets increase over time (updated yearly) based on population growth.

- Every time a new source is populated within the SourceArea, the number of households, restaurants and supermarkets of the populated source are deduced from those of SourceArea.

- SourceArea generates and dumps food waste at a generation rate.

10. SubGridCell:

- Spatial location is static.

- Initially populated with a number from an existing database.

- Sensitivity of a SubGridCell is pre-calculated as a function of number of people and their distance from the SubGridCell.

\subsubsection{Design concepts}

Emergence: The primary emergent behaviour of the ABM is the spatial, temporal and sizewise distribution of $A D$ operations within the study area. This emergence is partly a collective 
result of interaction of the two feasibility agents (DummyCollector and DummyPlant), with the Source agents.

Adaptation and Objectives: The model agents do not have any adaptive behaviour rules. However, some of the agents make their decisions based on an optimisation function, e.g. the DummyCollector identifies the next optimum collection area that will result in minimum travel distance for collection trucks while satisfying the feedstock demand for the next AD plant.

Sensing and Interaction: The model assumes free flow of information, e.g. DummyPlant has the information of acceptability for new Plants within the neighbourhoods around the collection area. The interaction between the main agents (Source, Collector and Plant) are primarily indirect through Main, DummyCollector, DummyPlant, SourceArea, GridCell and SubGridCell agents.

Stochasticity: The model requires a significant amount of data, which naturally has uncertainty and may not be available. Therefore, assumptions made in providing values for those model input parameters create some uncertainty. All the uncertainties in the model input parameters are accounted for in the model by randomising the values through a probability function.

\subsubsection{Input parameters}

Initialisation, temporal variation and input parameter uncertainty:

The model initialises the values of input parameters by randomly selecting a value within the relevant range in Baseline conditions, given in Table 2. The Baseline, Share \& Connect and Big \& Smart are initialised with the same randomly generated values at the beginning of the simulation year (2017). For the Baseline, the parameter values stay the same over the simulation period. The temporal change in the scenario parameter values is a function of the relevant initial (2017) value, the average value (Table 2) and the simulation period (2017 up to 2050).

A Monte Carlo simulation of 1000 runs was employed for each temporal variation to account for the input parameter uncertainties. Table 3 shows an example of how parameter values are initiated in the ABM for a single Monte Carlo run. For actual ABM simulations, the rows in Table 3 extend to year 2050 and the table is implemented 1000 times, each with a different randomly-selected initial value. 
Table 3: Example of input parameter initialisation and variation for a Monte Carlo run. $x$ and $n$ represent the limits of a Baseline parameter, $y$ is the average parameter for a scenario and $z$ is the randomly selected value in Baseline.

\begin{tabular}{|l|l|l|}
\hline & Baseline & Scenario \\
\hline Range & $x$ to $n ; n \geq \mathrm{x}($ Table 2) & NA \\
\hline Average & $\frac{x+n}{2}$ & $y$ (Average Values in Table 2) \\
\hline 2017 & Uniform $(\mathrm{x}, n)=z ; x \leq z \leq n$ & $z$ \\
\hline 2018 & $\mathrm{z}$ & $z+\frac{y-\frac{x+n}{2}}{2050-2017}$ \\
\hline
\end{tabular}

\section{$\underline{\text { AD diffusion }}$}

The future diffusion of $A D$ plants refers to the spatial dispersal of plants across $G B$, which has a population of 66 million and a total area of $209,331 \mathrm{~km}^{2}$. The initial (Baseline) population of the Plant was retrieved from ADBA (Thomas, 2017) and is shown by Figure 3. Diffusion is based on locations and amounts of food waste, generated in linear proportion to the population growth, AD capacity and probability mass functions ( $\mathrm{pmf}$ ). The plant capacity varies from micro ( $<1$ tonne/day), small (1-50 tonne/day), medium (50-150 tonne/day) to large ( $>150$ tonne/day). A feasibility search is run by the ABM to identify potential viable AD plant locations considering the relevant scenario-specific capacity and pmf values. The availability of food waste is the main limiting criterion for the AD diffusion.

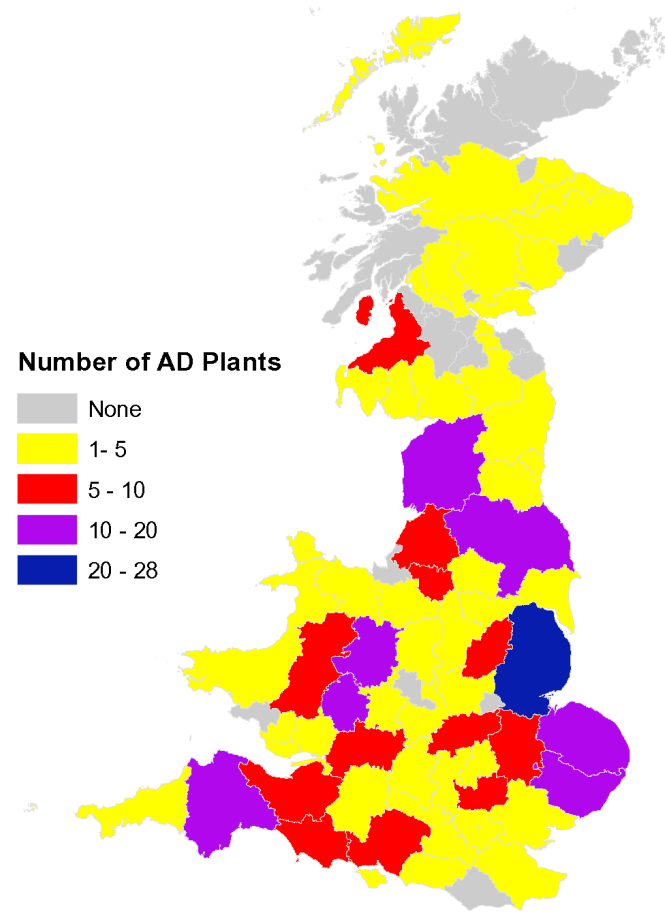

Figure 3: The total number of non-sewage AD Plants in Great Britain by county as of 2017. Generated based on Thomas (2017). 
Besides the global model parameters such as population growth rate, the initial agent populations (Collector, Plant, Source, Scenario, GridCell, SubGridCell) were generated using existing database tables. GridCell and SubGridCell agents define the spatial environment of the study area and the database tables for these agent populations were generated with some GIS pre-processing.

\subsubsection{Output parameter uncertainty}

Each model run generated 1000 output values, i.e. a value for each Monte Carlo run. Therefore, and in order to visualise each output parameter range, a probability density function (PDF) was plotted for each output using Equation 1.

PDF of Output Range $=\frac{\text { Histogram Output Parameter } \text { Values }}{N \times B W}$

$N=$ number or runs (1000) and BW is the bandwidth which has a value of one.

The PDF plots, as exampled in Section 3.1, indicated that the usual variation in each output had low probabilities for excessively low and large values. Hence, the median of each output range was used to plot the results, while error bars, in Section 3.2, reflected the minimum and maximum values of each output parameter, excluding the infrequent excessively low and large values.

\subsubsection{Variables and relevant functions}

The final outputs of the ABM and their relevant functions are summarised in Table 4. 
Table 4: ABM variables and their relevant functions, refer to Table 2 for relevant abbreviations.

\begin{tabular}{|c|c|c|c|c|}
\hline Parameter & Unit & Description & Relevant function & Notes \\
\hline $\begin{array}{l}\text { Total food } \\
\text { waste (TF) }\end{array}$ & $\begin{array}{l}M \\
\text { ton }\end{array}$ & $\begin{array}{l}\text { Total food waste generated } \\
\text { to date within the Source } \\
\text { Area }\end{array}$ & $\begin{array}{l}\mathrm{TF}=\mathrm{DF} * \text { Duration } \\
{ }^{*} 365\end{array}$ & $\begin{array}{l}\text { FW is the daily food } \\
\text { waste produced from } \\
\text { households, } \\
\text { restaurants and } \\
\text { supermarkets. }\end{array}$ \\
\hline $\begin{array}{l}\text { Recycled food } \\
\text { waste (RF) }\end{array}$ & $\begin{array}{l}M \\
\text { ton }\end{array}$ & $\begin{array}{l}\text { food waste used for the AD } \\
\text { process }\end{array}$ & $\mathrm{RF}=\mathrm{TF}{ }^{*}$ baseRR & \\
\hline $\begin{array}{l}\text { Dumped food } \\
\text { waste (DF) }\end{array}$ & $\begin{array}{l}M \\
\text { ton }\end{array}$ & $\begin{array}{l}\text { Food waste that was not } \\
\text { recycled }\end{array}$ & $\mathrm{DF}=\mathrm{TF}-\mathrm{RF}$ & \\
\hline $\begin{array}{l}\text { Produced } \\
\text { Biogas (BG) }\end{array}$ & $\begin{array}{l}M \\
m^{3}\end{array}$ & $\begin{array}{l}\text { The total amount of biogas } \\
\text { from the AD process. }\end{array}$ & $\begin{array}{l}\mathrm{BG}=\mathrm{TF} * \\
\mathrm{BG} \_ \text {coeff }\end{array}$ & \\
\hline $\begin{array}{l}\text { Water } \\
\text { coefficient } \\
\text { (W_coeff) }\end{array}$ & $1 / \mathrm{kg}$ & $\begin{array}{l}\text { Specific water consumption } \\
\text { for food waste digestion }\end{array}$ & & \\
\hline $\begin{array}{l}\text { Consumed } \\
\text { water (W) }\end{array}$ & $\begin{array}{l}M \\
m^{3}\end{array}$ & $\begin{array}{l}\text { The amount of water fed into } \\
\text { the } A D \text { process. }\end{array}$ & $\mathrm{W}=\mathrm{TF}{ }^{*} \mathrm{~W} \_$coeff & \\
\hline $\begin{array}{l}\text { Produced } \\
\text { Digestate (D) }\end{array}$ & $\begin{array}{l}M \\
\text { ton }\end{array}$ & Digestate output rate & $\mathrm{TF}+\mathrm{W}-\mathrm{BG} * 1.15$ & \\
\hline $\begin{array}{l}\text { Produced } \\
\text { Landfill (LF) }\end{array}$ & $\begin{array}{l}M \\
\text { ton }\end{array}$ & $\begin{array}{l}\text { The amount of contaminants } \\
\text { (during the processing of the } \\
\text { food waste) that build } \\
\text { landfills. Larger LF results in } \\
\text { negative social impact. }\end{array}$ & $L F=T F^{*}\left(F \_c o e f f-1\right)$ & \\
\hline $\begin{array}{l}\text { Total Distance } \\
\text { (TD) }\end{array}$ & $\mathrm{km}$ & $\begin{array}{l}\text { Total distance travelled by } \\
\text { trucks; applies for } \\
\text { transporters and collectors }\end{array}$ & $\begin{array}{l}T D= \\
\text { Av_round_trip_dist } \\
{ }^{*} \text { No of trips }\end{array}$ & \\
\hline Total $\mathrm{CO}_{2}(\mathrm{TC})$ & $\begin{array}{l}M \\
\text { ton }\end{array}$ & $\begin{array}{l}\text { Total } \mathrm{CO}_{2} \text { produced from } \\
\text { transporting food waste }\end{array}$ & $\begin{array}{l}\mathrm{TC}=\mathrm{TD}^{*} \text { Truck } \\
\text { Emission Rate }\end{array}$ & \\
\hline $\begin{array}{l}\text { Total } \\
\text { Transportation } \\
\text { Costs (TTC) }\end{array}$ & $M £$ & $\begin{array}{l}\text { Total costs of transportation } \\
\text { including fuel \& driver } \\
\text { salaries; applies for } \\
\text { transporters and collectors } \\
\text { Total number of AD plants. }\end{array}$ & $\mathrm{TTC}=\mathrm{TD}{ }^{*} \mathrm{KOC}(\mathrm{tt})$ & $\begin{array}{l}\text { tt stands for truck } \\
\text { type }\end{array}$ \\
\hline $\begin{array}{l}\text { Number of AD } \\
\text { Plants (NoP) }\end{array}$ & NA & $\begin{array}{l}\text { This applies to active and } \\
\text { inactive micro, small, } \\
\text { medium \& large plants. }\end{array}$ & fun(pmf) & \\
\hline $\begin{array}{l}\text { Total Capital } \\
\text { Costs (TCC) }\end{array}$ & $M £$ & $\begin{array}{l}\text { Total costs of installing AD } \\
\text { plants }\end{array}$ & $\begin{array}{l}\text { TCC = Sum } \\
\left(\mathrm{SCC}(\mathrm{pc})^{*}\right. \\
\operatorname{NoP}(\mathrm{pc}))^{*}\end{array}$ & $\begin{array}{l}\text { pc stands for plant } \\
\text { category }\end{array}$ \\
\hline $\begin{array}{l}\text { Total } \\
\text { Operational } \\
\text { Costs (TOC) }\end{array}$ & $M £$ & $\begin{array}{l}\text { Total costs of operating the } \\
A D \text { plants and transportation } \\
\text { costs }\end{array}$ & $\begin{array}{l}\text { TOC }=\text { Sum } \\
\left(\mathrm{AOC}(\mathrm{pc})^{*}\right. \\
\mathrm{NoP}(\mathrm{pc}))^{+}+\mathrm{TTC}\end{array}$ & \\
\hline $\begin{array}{l}\text { Social } \\
\text { Disturbance } \\
\text { (SD) }\end{array}$ & NA & $\begin{array}{l}\text { Disturbance to the public } \\
\text { resulting from installing AD } \\
\text { within a close vicinity } \\
\text { (sen.thres) of residential } \\
\text { areas }\end{array}$ & $\begin{array}{l}\mathrm{SD}=\text { fun }(\mathrm{NoP} \\
\text { sens.thres) }\end{array}$ & $\begin{array}{l}\text { sens.thres is the } \\
\text { threshold distance } \\
\text { from residential } \\
\text { areas, that's socially } \\
\text { acceptable by the } \\
\text { public to install AD } \\
\text { plants. }\end{array}$ \\
\hline
\end{tabular}




\subsection{Quantifying environment, social and economic impacts}

This section explains how the above impacts are quantified and visualised. For a fair comparison and to highlight the potential of $A D$ in different future alternatives, each scenario was normalised and benchmarked to the Baseline.

The environmental impact is the relative $\mathrm{CO}_{2}$ produced from each scenario in comparison to the Baseline as expressed by Equation 2.

Environmental Impact $=\frac{T C(s)-T C(b)}{T C(b)}$

$T C$ is total carbon emissions computed by the ABM using the relevant function in Table 4, $s$ and $b$ stand for scenario and baseline respectively.

The economic impact was quantified based on capital and operational costs and costs avoided (CA). Financial incentives were not considered in this study since there is a lack of reliable consistent data. Equation 3 shows the formula used for computing the Economic Impact.

Economic Impact $=\frac{C A(s)-C A(b)}{C A(b)}-\frac{T O C(s)+T C C(s)-(T O C(b)+T C C(b))}{T O C(b)+T C C(b)}$

CA is costs avoided, TOC is the total operational costs, TCC is the total capital costs.

Social impact in the broader sense refers to a host of factors related to health and wellbeing, including; distribution of welfare, sense of community, mobility, recreational value, job creation, perception of nature and cultural environment, aesthetics, safety, to name a few. There can be positive or negative social impacts of technologies on each of these factors. For the purposes of this paper, the social impact of $A D$ technology is identified in terms of the disturbance it causes to households, abated by the reductions in landfill. The disturbance was selected as a key factor due to evidence of NIMBYism ('not in my back yard' - meaning undesirable to be close) for such plants in urban areas. This arises due to the odour associated with $A D$ operations, transportation and the negative visual impact.

The normalised social impact is calculated by Equation 4 .

Social Impact $=\frac{S D(s)-S D(b)}{S D(b)}+\frac{L F(s)-L F(b)}{L F(b)}$

$S D$ is the social disturbance, as defined in Table 4. LF is the total produced landfill from $A D$ implementation.

\subsection{Quantifying the impacts on the water-energy-food nexus}

The WEF impacts were quantified in a similar manner to that of the environmental, social and economic impacts. Equations 5, 6 \& 7 compute the relative impacts on the water, energy and food areas respectively.

Impact on Water $=\frac{W(s)-W(b)}{W(b)}$

$W$ is the total water consumed in $A D$ operation. 
Impact on Energy $=\frac{B G(s)-B G(b)}{B G(b)}$

$B G$ is the amount of produced biogas.

Impact on Food $=\frac{R F(s)-R F(b)}{R F(b)}+\frac{D(s)-D(b)}{D(b)}$

$R F$ is the total amount of recycled food waste while $D$ is the total produced digestate.

The impacts mentioned in sections 2.5 and 2.6 were computed for years 2025, 2033, 2040 \& 2050 to present the temporal variation of potential AD implications.

\section{Results}

Before illustrating the ABM results, this section explains how the ABM outputs were distributed using probability density function (PDF) plots.

\subsection{PDF example of an output range}

Figure 4 shows the PDF of the number of active medium plants which was the typical distribution for an output parameter and hence, used as an example to determine the realistic minimum and maximum output parameter values. Observing the PDF plots for the output parameters in the baseline and the two scenarios, it was noticed that excessively low and large values occur at a probability (vertical axis) values that is around $25 \%$ of the maximum PDF. For example, the PDF of the number of active medium plants (Figure 4) varied from close to zero values (very low frequency) and 0.058 (highest frequency), while values below 40 and above 70 have much lower frequencies than the rest of the range. Therefore, and in order to present realistic results, output parameter values that have PDF less than $25 \%$ of the maximum parameter PDF were neglected. 


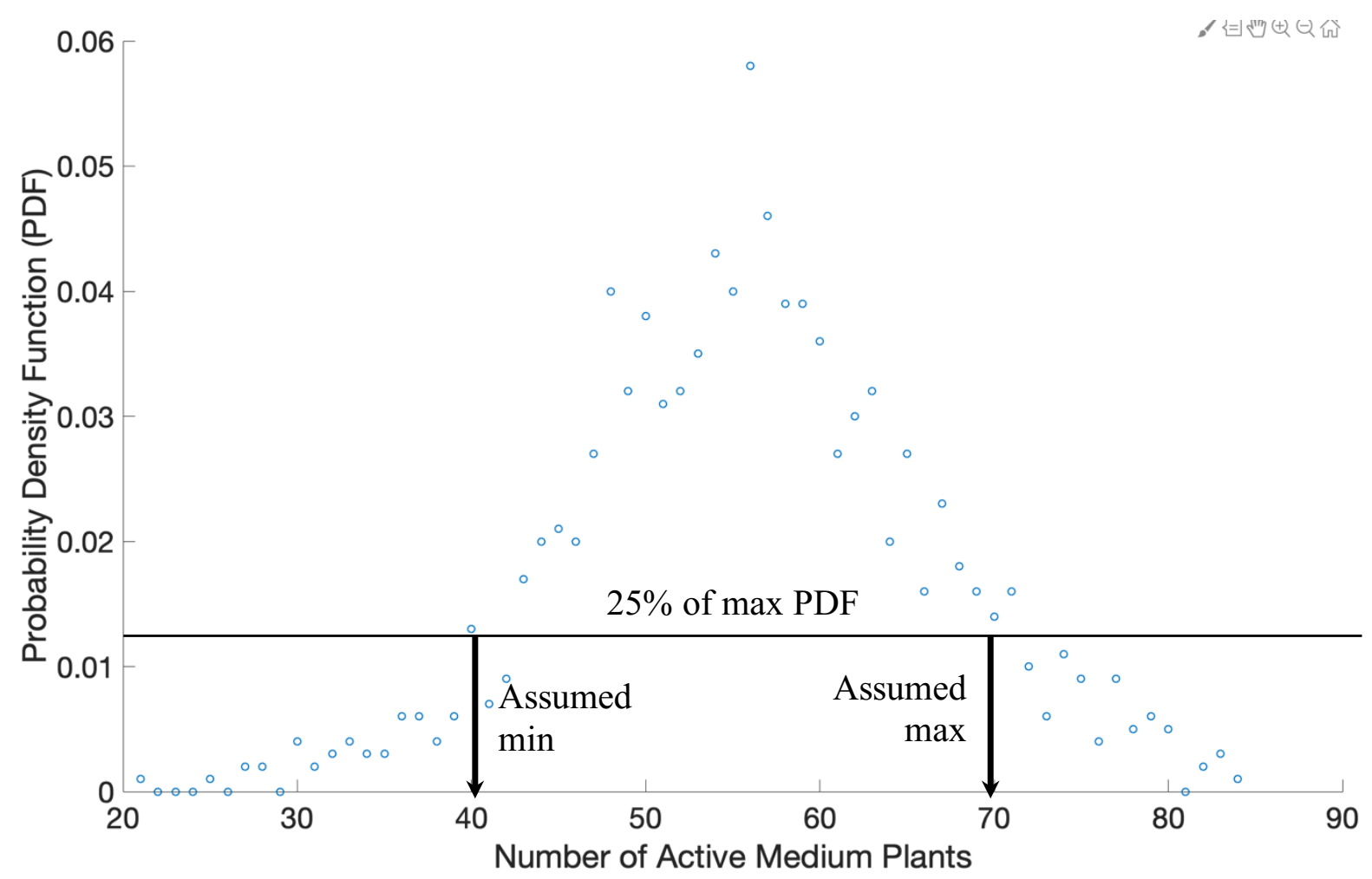

Figure 4: Probability Density Function (PDF) for an output parameter range; the number of active medium plants in Big \& Smart.

\subsection{Simulation outputs for 2050}

Figures in this section were created to plot the accumulated model outputs, between 2017 \& 2050.

Number of AD plants

The number of $A D$ plants reflects the nature of the scenario. Share \& Connect presented the highest number of $A D$ plants mainly due to the creation of more micro plants. Referring to Share \& Care description in Table $1, A D$ is a widespread technology that locals invest in and is flexible for installation at household scales (micro). Figure 5 shows the accumulated number of active and inactive $A D$ plants (Section 2.4.4) in the baseline and the two simulated scenarios. 


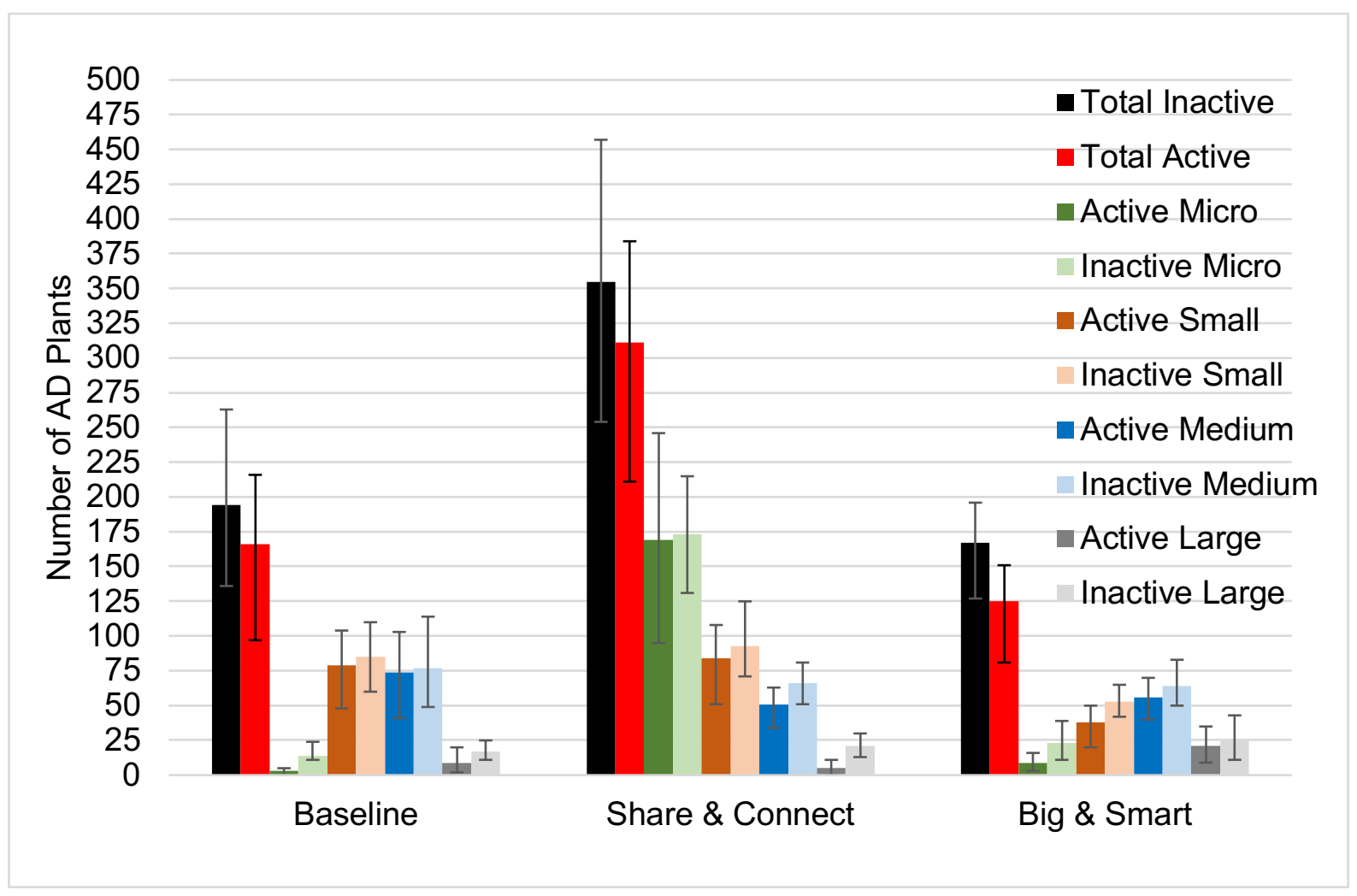

Figure 5: Number of active and inactive AD plants for the period between 2017 \& 2050.

Total costs

Capital costs were relatively similar in the two scenarios and the Baseline. This is expected when considering the specific capital costs for more micro plants in Share \& Connect and more large plants in Big \& Smart.

The operational cost for Share \& Connect was $34 \%$ less than the Baseline. This is due to the majority of $A D$ plants in Share \& Connect being active micro and small which have lower operational costs than those of large plants in the Baseline. Big \& Smart showed slightly (5\%) higher total operational costs than the Baseline, because the number of large AD plants in Big \& Smart is double of that in Baseline.

Transportation costs for Share \& Connect were the lowest. Despite the same transportation and collection vehicle costs in Share \& Connect as that of the Baseline, less distances are travelled in Share \& Connect since most of the AD plants are installed locally. On the other hand, Big \& Smart has the largest number of AD plants that are located far from the feedstock sources, hence the larger transportation costs. The total accumulated capital and operational costs are shown in Figure 6. 


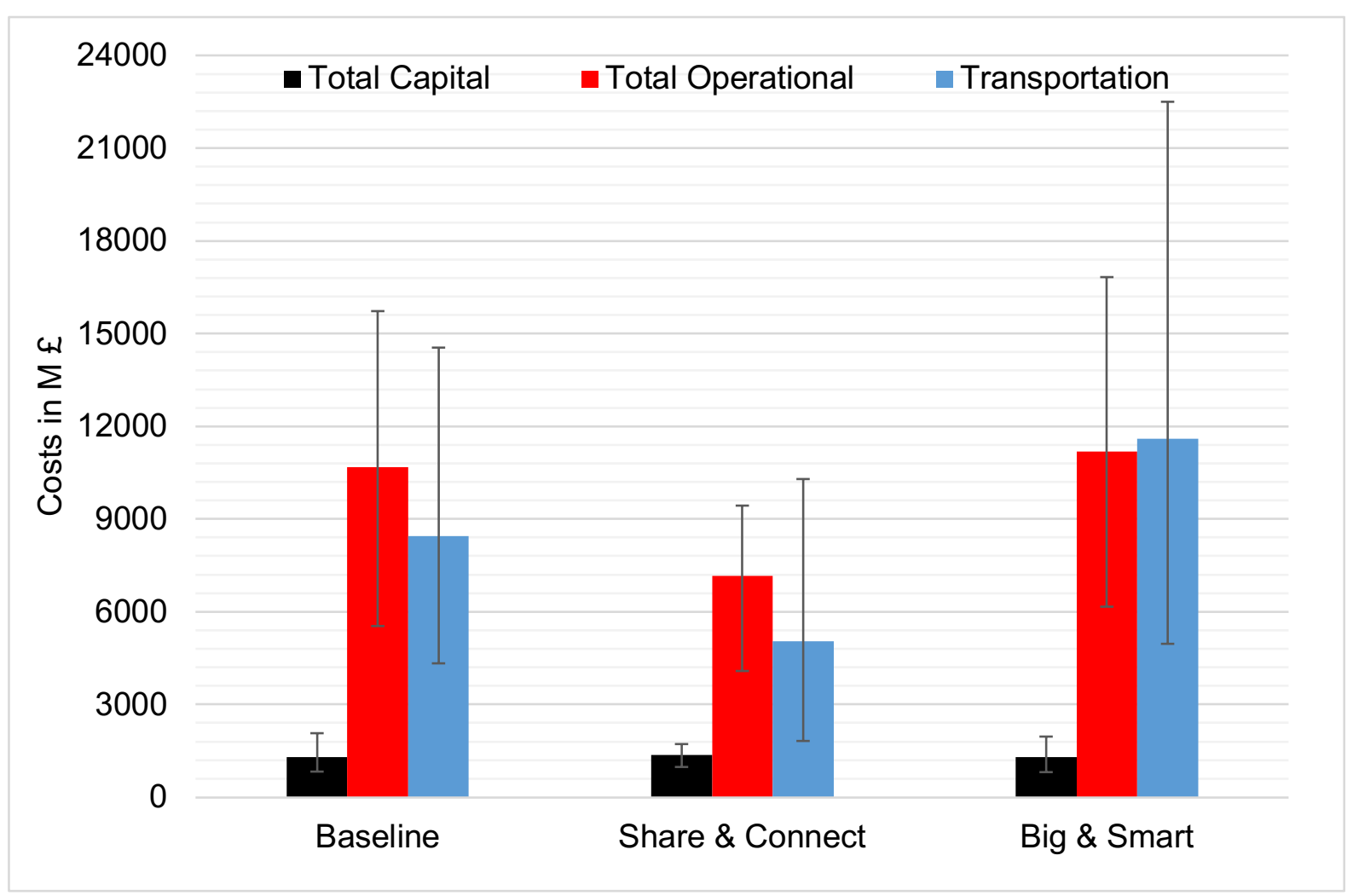

Figure 6: Total capital, operational and transportation costs between 2017 \& 2050.

Food waste

Total food waste was the highest in the Baseline, followed by Big \& Smart and then Share \& Connect scenarios which reflects the rates for household, restaurants and supermarket food waste generation. The recycled food waste for Big \& Smart (150 Megatonnes) was slightly higher than that for the Baseline (133 Megatonnes), thanks to the highest recycling ratio in Big \& Smart. Figure 7 shows the food waste produced in Baseline and the two modelled scenarios. 


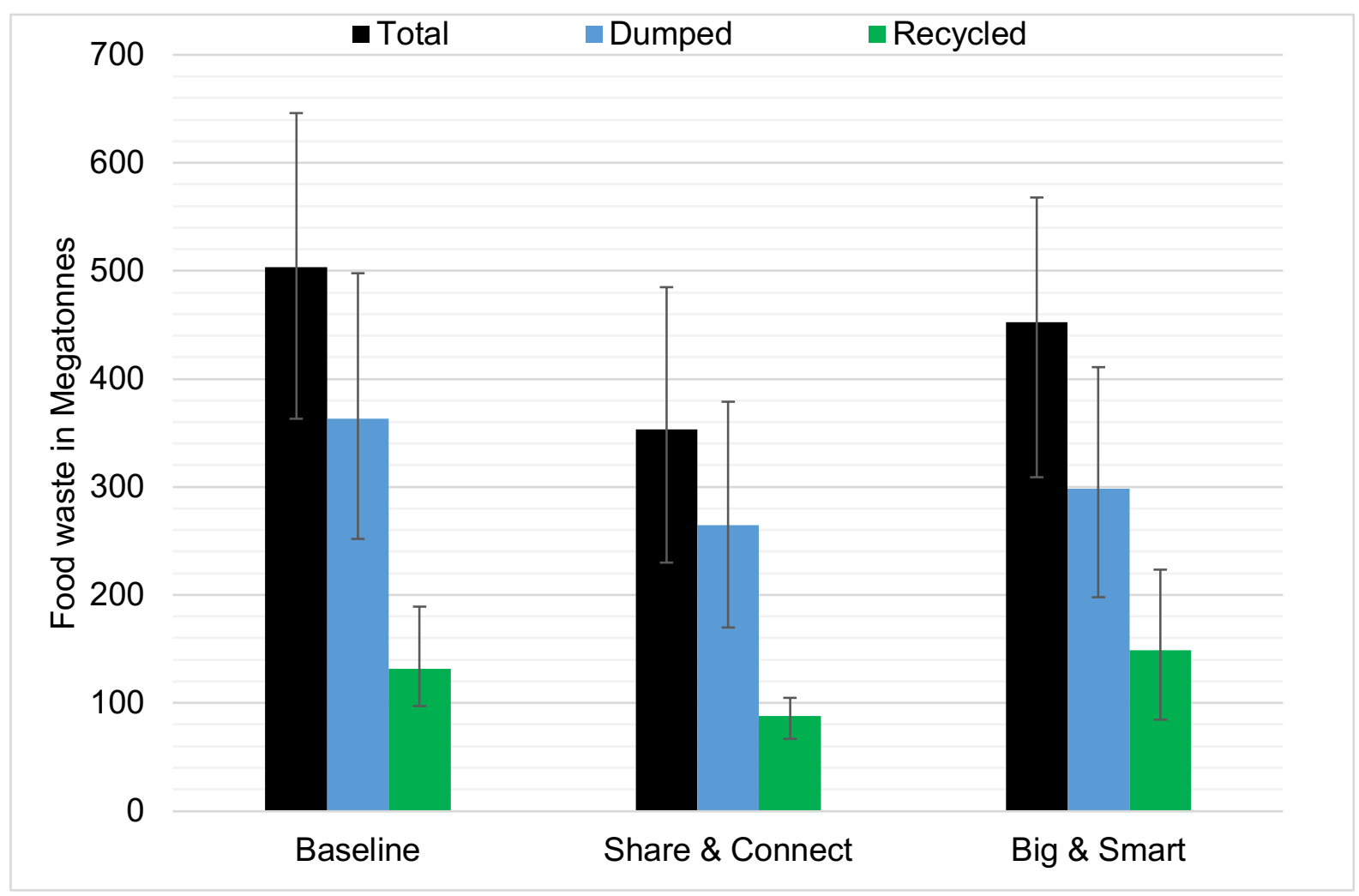

Figure 7: Total food waste produced between 2017 \& 2050.

Produced biogas

Across the scenarios, Biogas production showed similar variations to those of food waste, with Big \& Smart showing slightly (4\%) higher quantity than the Baseline. The higher recycled food waste in Big \& Smart generated more biogas in comparison to the Baseline. Figure 8 shows the total accumulated biogas produced for the Baseline and the two simulated scenarios. 


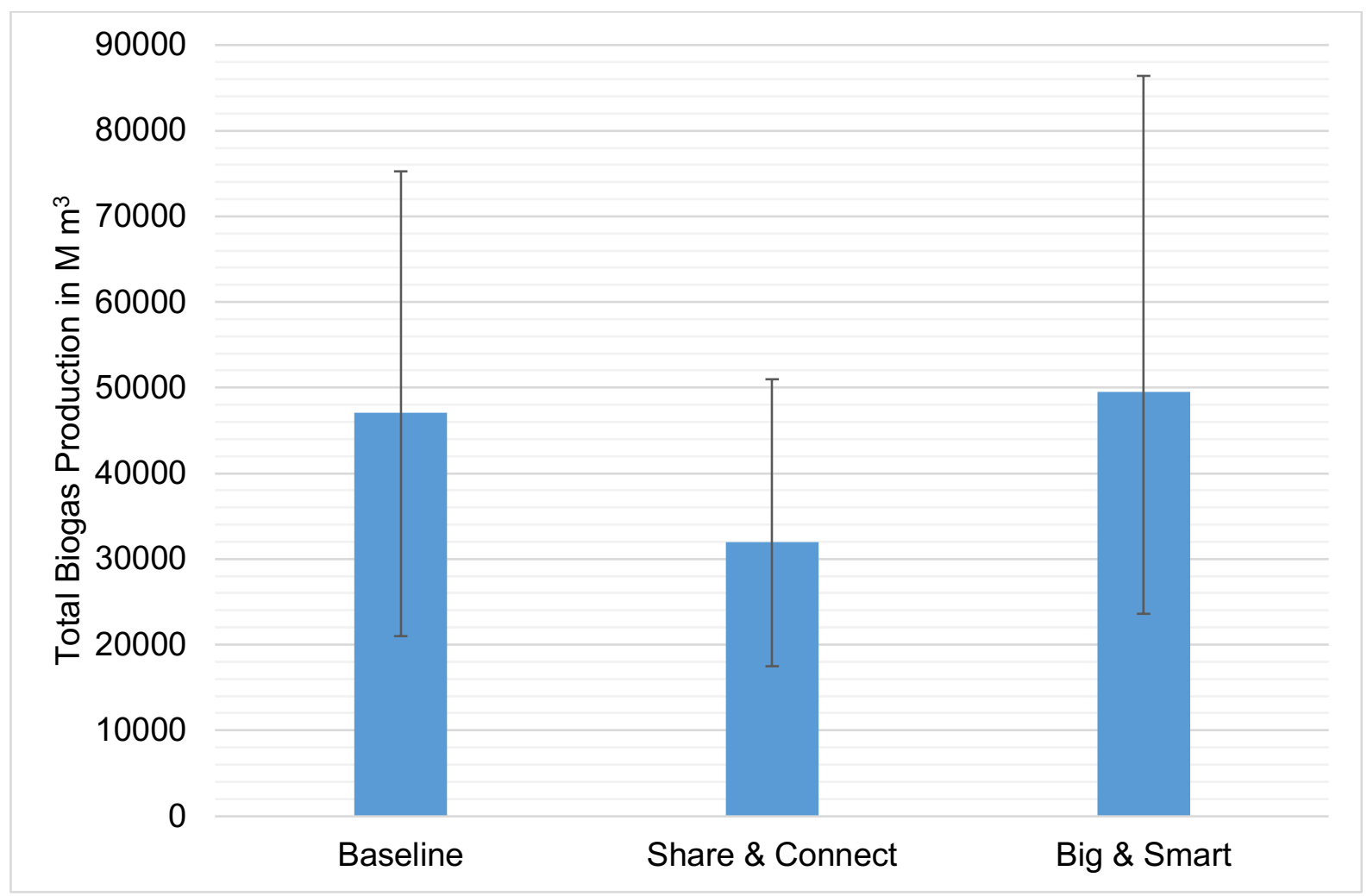

Figure 8: Total biogas produced between 2017 \& 2050.

\section{Produced digestate}

The variation in digestate production was similar to that of biogas across the Baseline and the two scenarios, which is expected since it is a function of the feedstock. Big \& Smart, which assumes improved AD digestate quality due to R\&D work, presents a greater potential for agricultural benefits than Share \& Connect because of the $56 \%$ more digestate production in the former scenario. The total produced digestate is shown in Figure 9. 


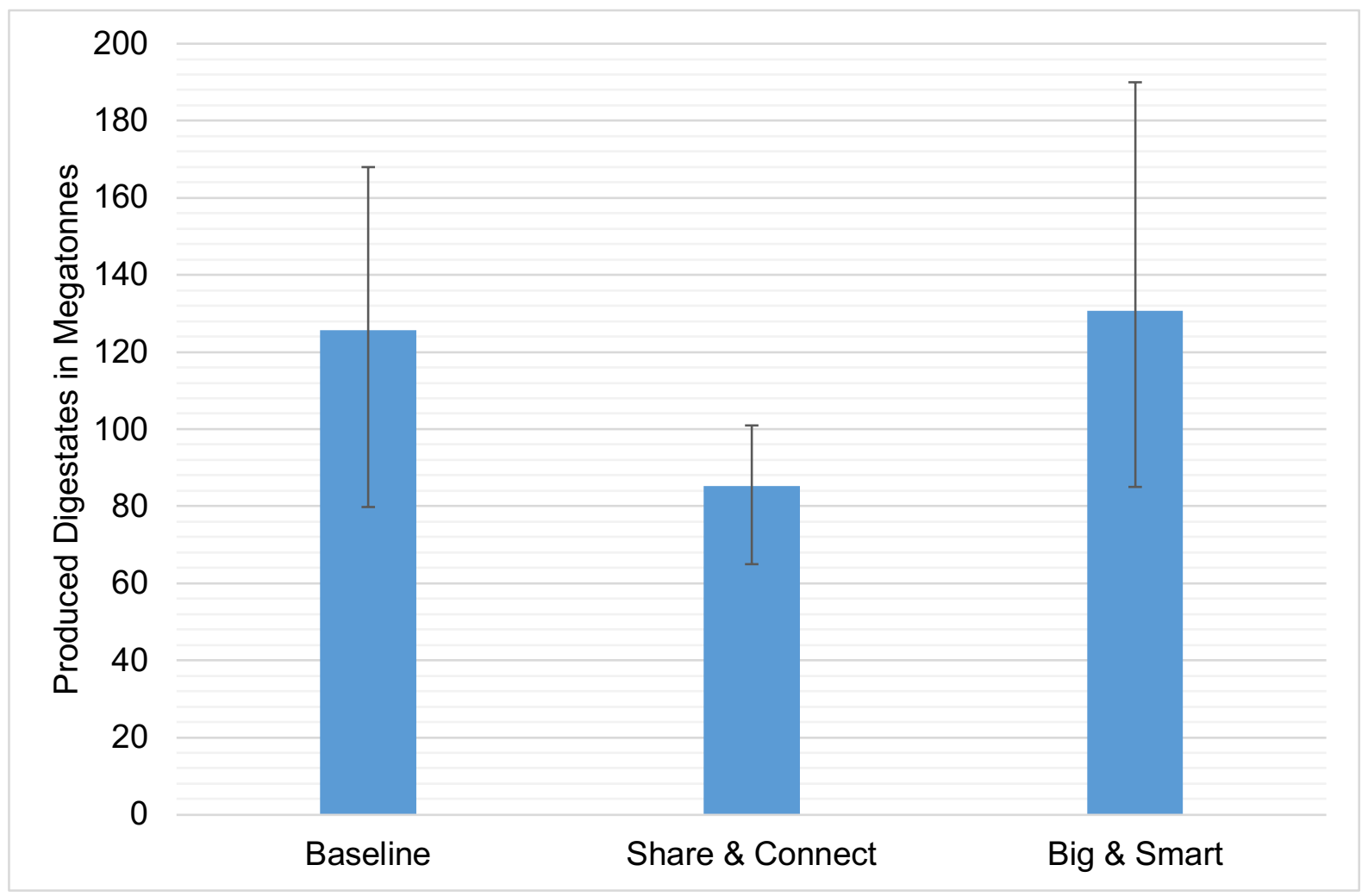

Figure 9: Total produced digestate between 2017 \& 2050.

Water consumption

The pattern of water consumption for AD operation, shown by Figure 10, is similar to that of biogas production, which is expected due to the proportionality relationship between the two parameters.

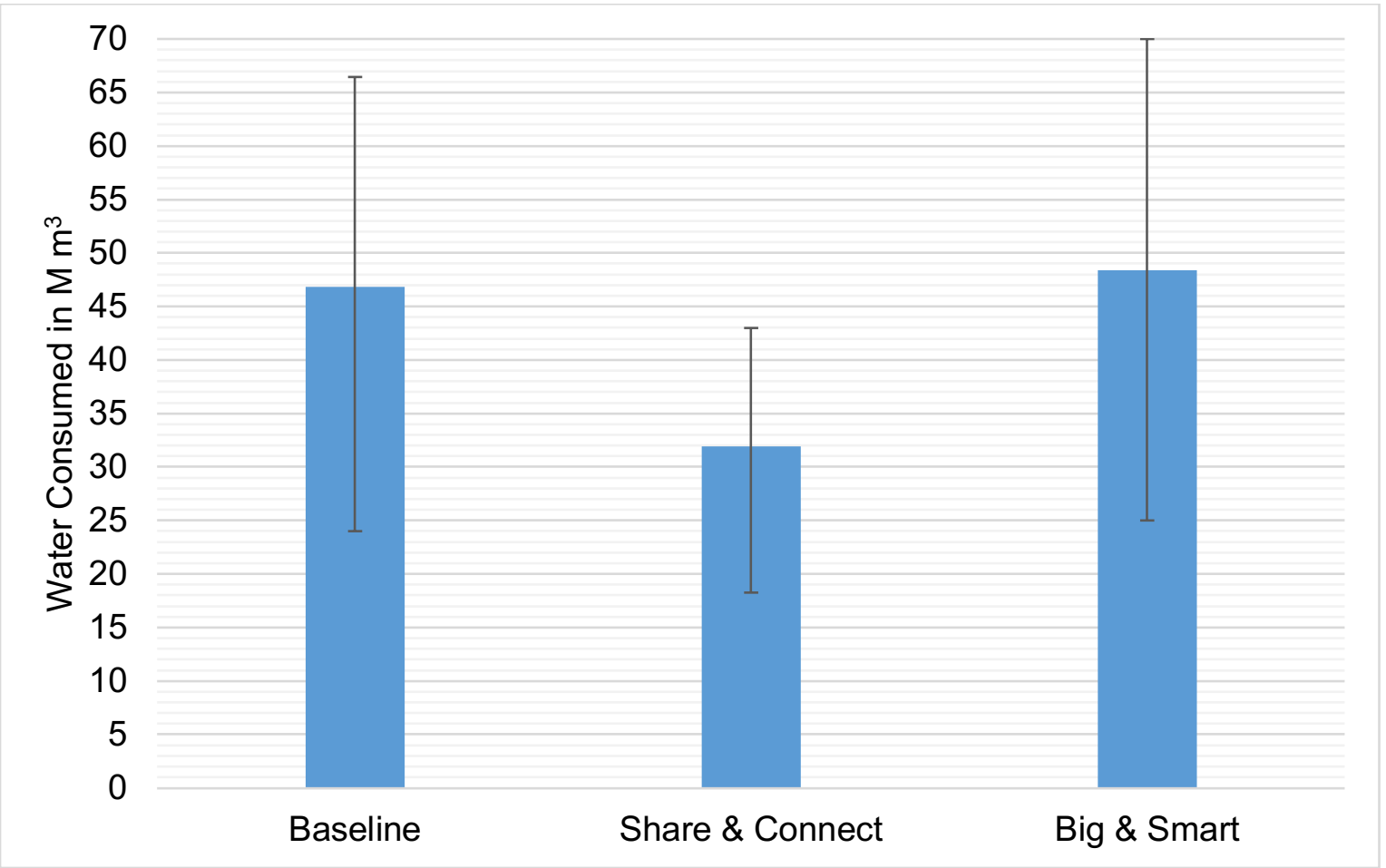

Figure 10: Total water consumed, by the AD plant, between 2017 \& 2050. 


\section{Carbon emissions}

The relative carbon reductions resulting from transporting and collecting food waste, in relation to the Baseline, were $65 \%$ and $10 \%$ for Share \& Connect and Big \& Smart respectively. The large reduction in Share \& Connect is due to the local micro and small plants which minimise transportation. The $\mathrm{CO}_{2}$ reduction in Big \& Smart is due to the use of electric transportation and collection vehicles that are fuelled by decarbonised sources, whose impact is signified by the longer distances travelled in Big \& Smart. The pattern of carbon emissions across the Baseline and the two scenarios is correlated with transportation costs (Figure 6) which is expected since both parameters reflect vehicle fuel consumption. Figure 11 shows the total carbon emissions produced from transportation in Baseline and the two scenarios.

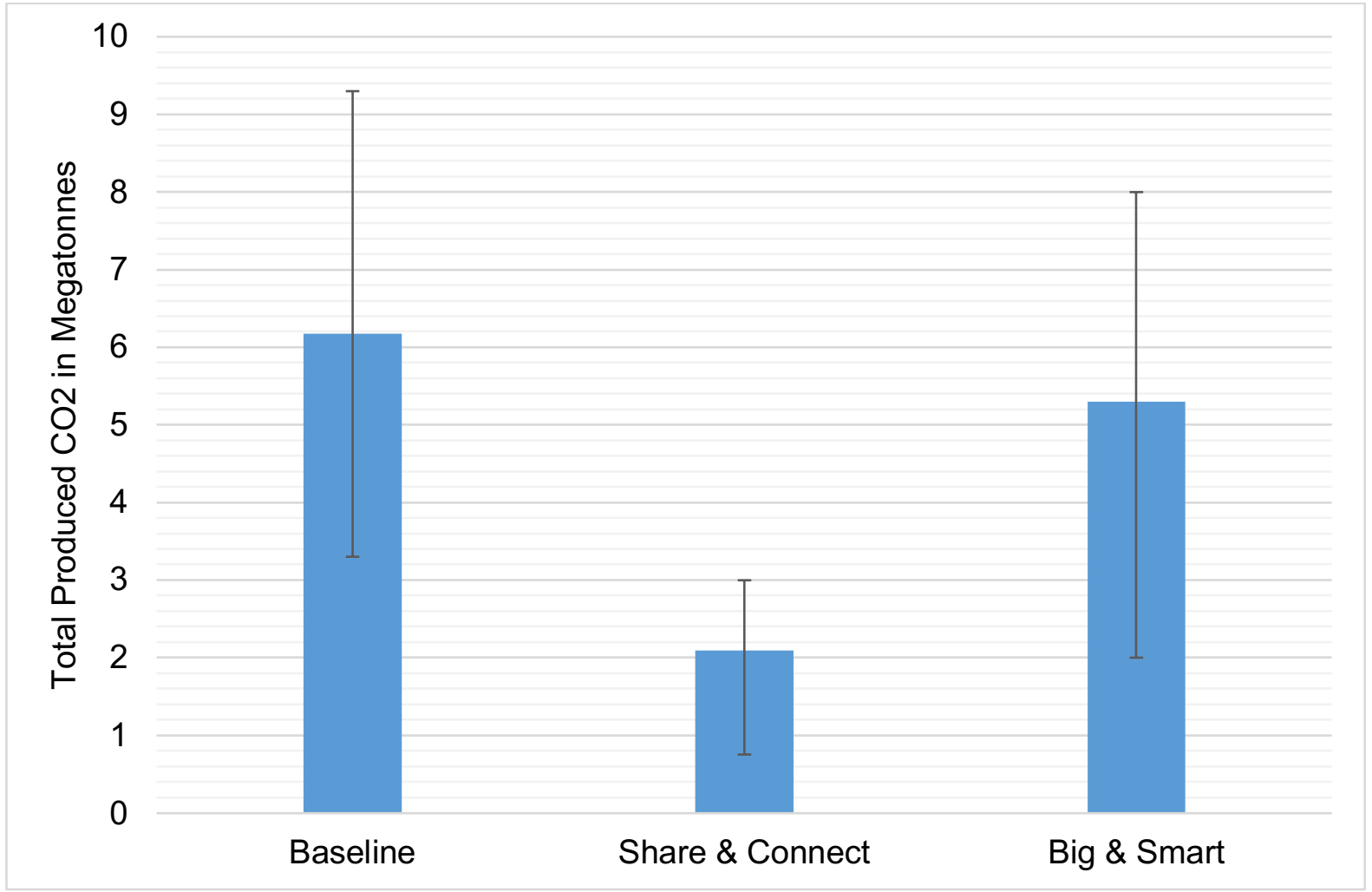

Figure 11: Total transportation $\mathrm{CO}_{2}$ emissions between 2017 \& 2050.

\subsection{Simulated impacts for $2025,2033,2040 \& 2050$}

Share \& Connect showed the worst social impact since the large number of local (micro) AD plants causes greater disturbance to the public than Big \& Smart. However, Share \& Connect showed the least landfill production, which slightly mitigates its overall adverse social impact. Figure 12 shows the environmental, social and economic impacts of Share \& Connect and Big \& Smart, while Figure 13 shows the impacts on the WEF nexus for the two scenarios. 

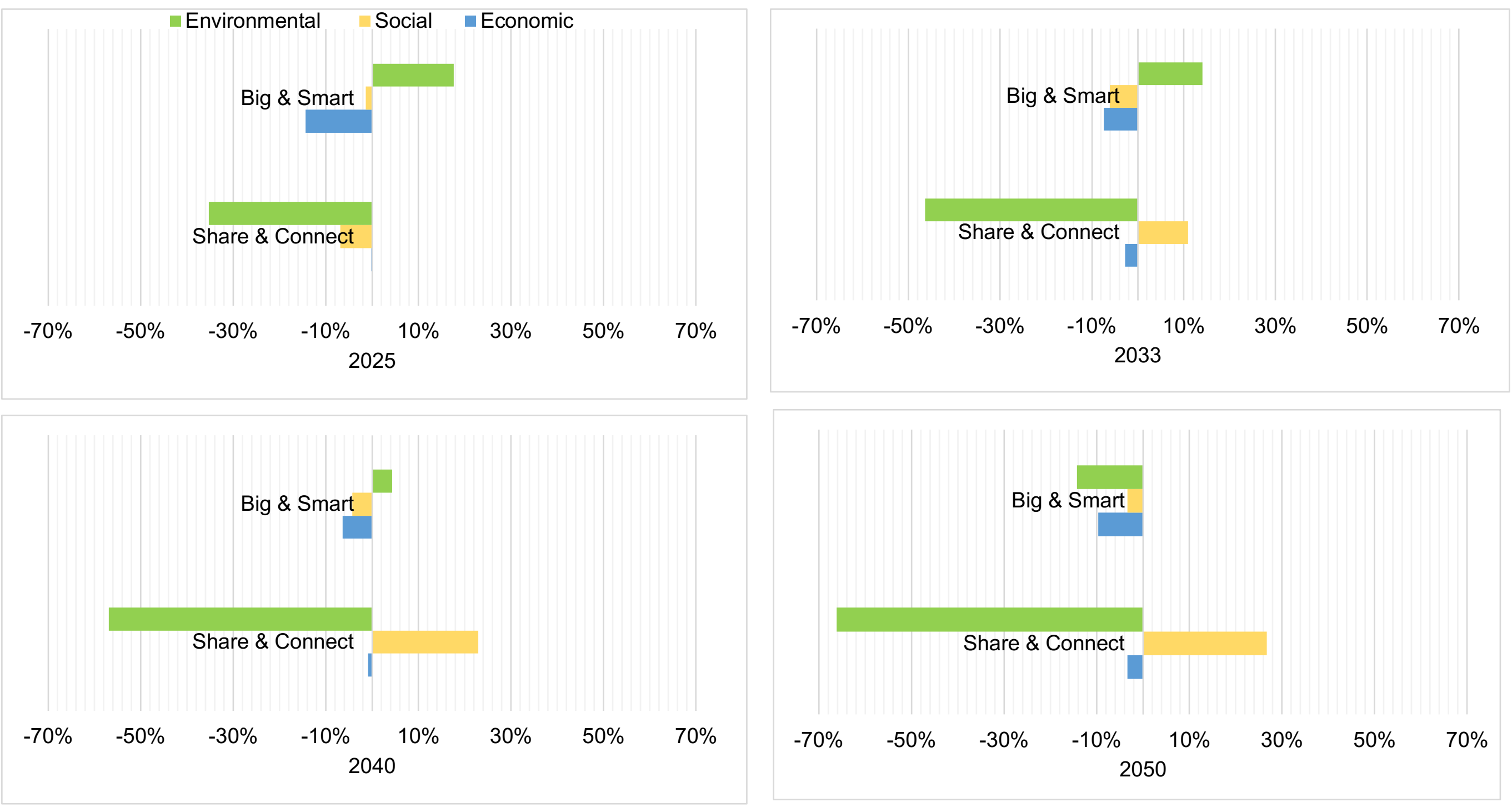

Figure 12: Variation of Environmental, Social and Economic impacts in years 2025, 2033, 2040 \& 2050 in relation to Baseline (Equations 2, 3 \& 4). Negative percentages indicate improvements compared to Baseline. 

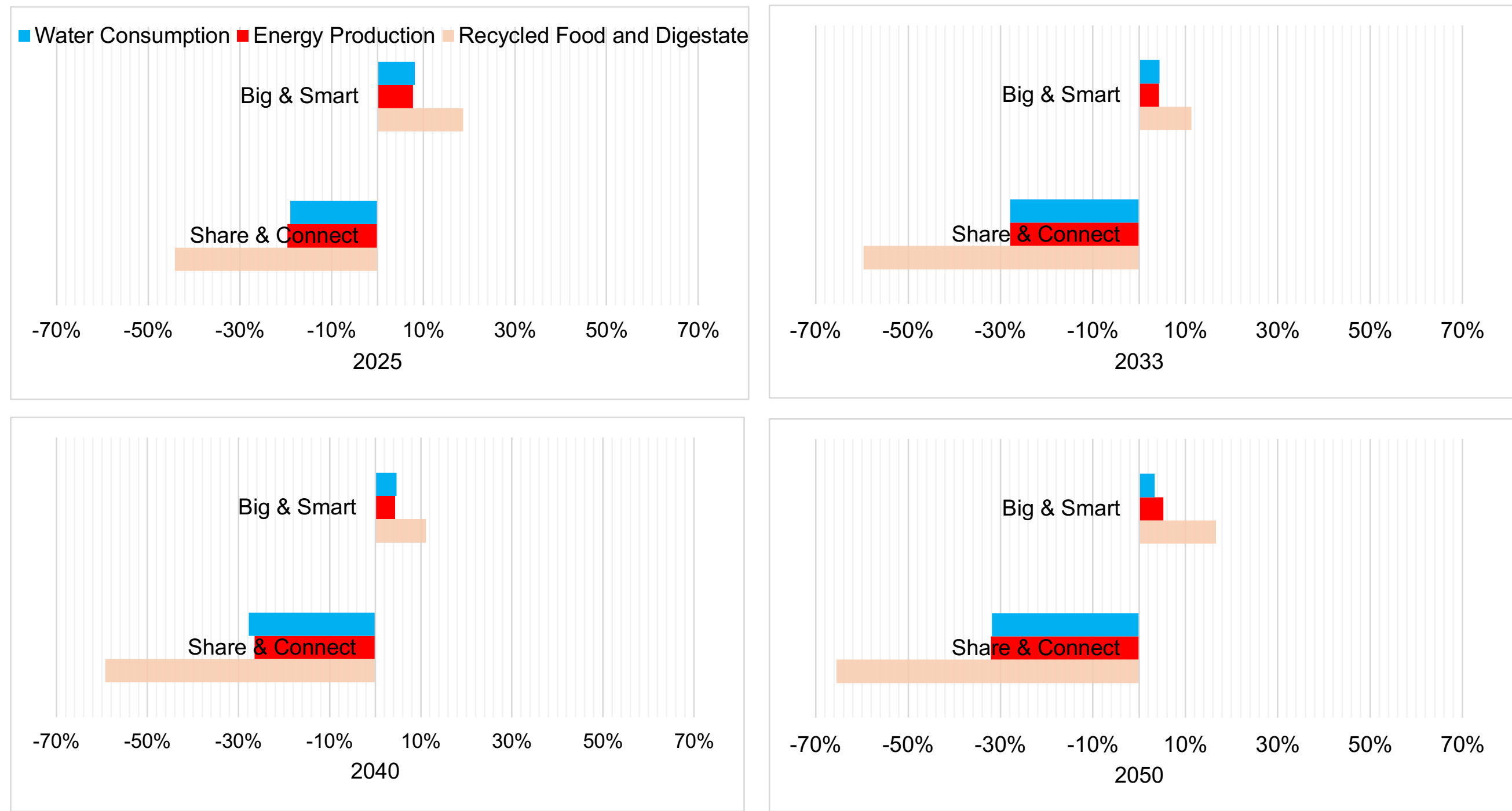

Figure 13: Variation of impacts on the Water Energy Food nexus in years 2025, 2033, 2040 \& 2050 compared to Baseline (Equations 5, 6 \& 7). Negative percentages indicate lower values than Baseline. 


\subsection{Output uncertainty (error bars)}

Transportation costs and Biogas production showed high uncertainties that reached almost twice the median value (Figure 6 and Figure 8, respectively), because of the difficulty in acquiring real data. Although the household food waste showed the largest variation in its input parameter, it was combined with other sources (supermarkets and restaurants) and hence reduced the uncertainties in the generated, recycled and dumped food wastes. Accounting for output parameter uncertainty through PDF (Section 3.1) has produced consistent variations in output parameters, and therefore minimised the overlapping of uncertainty between the Baseline and the two modelled scenarios.

\section{Discussion}

\subsection{Overall performance of ABM}

The ABM has quantified the potential benefits of future AD implementation based upon stated assumptions and input values. Variations in model output values between the Baseline and the two scenarios broadly reflect the constraints and opportunities provided by alternative future pathways, such as differences in food waste availability.

\subsection{Impacts on the environmental, social and economic aspects}

The impacts of future AD implementation are interlinked, and the ABM shows a compromise in terms of the environmental, social and economic benefits when evaluating the potential value and viability of $A D$ deployment in a particular scenario. Big \& Smart always makes the highest positive contribution to economic benefits across all the simulated years, whilst Share \& Connect produces much less carbon and hence results in a positive environmental impact. Big \& Smart starts with a negative impact on the environment in 2025, 2033 and 2040, i.e. more $\mathrm{CO}_{2}$ emissions than the Baseline, which then drops in 2050 to result in emissions' reduction of $14 \%$. This is mainly due to the assumed increase in the use of renewable transportation fuels, which also influences the reduction in the truck operational costs.

A major drawback of Share \& Connect is the undesirable social impact, caused by the reduced acceptability of $A D$ diffusion in residential areas. It was assumed that people's perceptions towards $A D$ installations in residential areas is constant in all simulations. This was essential for a fair comparison between the Baseline and the scenarios along the modelled years. However, one may argue that this perception may change over time as residents become more familiar with the $A D$ technology and more aware of its eco-environmental benefits, particularly in Share \& Connect where people invest directly in the technology. Greater social acceptability would have increased the quantity of $A D$ implementation, and the proportion of smaller plants leading to better economic and social impacts.

Although Big \& Smart results in more total costs by 2050 than the Baseline and Share \& Connect (by $5 \%$ and $35 \%$ respectively), it creates more potential for processing recycled food and produces more digestate than the Baseline (by 17\%) and much more than Share \& Connect (by $85 \%$ ). Anaerobic digestion digestate may reduce farming fertiliser costs but this is not accounted for in the model.

The ABM predicts $34 \%$ more energy production in the Big \& Smart scenario than Share \& Connect in 2050 . Considering a biogas calorific value of $23 \mathrm{MJ} / \mathrm{m}^{3}$ (SGC, 2012), it was 
estimated that by 2050, Baseline, Big \& Smart and Share \& Connect can produce 266,000 , 176,000 and $276,000 \mathrm{GWh}$ respectively, by CHP engines (around $35 \%$ electricity $\& 50 \%$ heat (Banks, et al. (2011)).

Despite Share \& Connect having the lowest total costs, Big \& Smart is more economical, over the simulation period, when accounting for the costs avoided (Figure 12). Between 2017 and 2050, utilising electricity and heat from CHP engines can result in avoiding significant costs of energy produced from conventional sources. Potential savings in 2050 were estimated to be $£ 27,000, £ 18,000$ and $£ 28,000$ billion for the Baseline, Share \& Connect and Big \& Smart respectively. This can have consequences for grid level energy production and may delay or avoid expensive grid reinforcement. In case of biogas injection to the gas grid, $£ 10,900, £ 7,200$ and $£ 11,300$ billion can be avoided in Baseline, Share \& Connect and Big \& Smart respectively. The above estimates were based on $19 \mathrm{p} / \mathrm{kWh}$ and $4 \mathrm{p} / \mathrm{kWh}$ for electricity and gas respectively, which explains the lower costs avoided in the biogas injection option. However, the costs associated with CHP plant and grid connection points were not considered, hence the estimates above are optimistic.

Whilst Share \& Connect appears to be a promising scenario for AD from an environmental perspective, there is a trade-off with its economic implications. The challenge is that there is no universal metric to compare economic, social and environmental impacts, and adequately assess the trade-off.

\subsection{Impacts on the WEF nexus}

There are some clear linearities on multi-sector impacts. The ABM highlights a clear trade-off between water consumption and energy produced: the more biogas (energy) produced, the more water consumed as noticed with Big \& Smart. On the other hand, the less food waste available, e.g. in Share \& Connect, the less energy produced and hence the less AD digestate generated, which can be considered a negative impact on the food sector since the digestate is assumed to be utilised for farming purposes. Share \& Connect demonstrates a positive influence on the reduction of water consumption compared to the Baseline (by up to $32 \%$ ) and Big \& Smart (by up to $36 \%$ ), which is expected since the amount of food waste processed for $A D$ is much lower than Big \& Smart and the Baseline. Implementing AD in a Share \& Connect scenario may therefore present an attractive option when severe water scarcity is a serious urgent matter. The patterns of the impacts on the WEF sectors have been consistent across the simulated years; where Big \& Smart always showed the largest potential for energy production.

\subsection{Invisible impacts and ABM limitations}

Positive impacts may accrue for the economy, since food waste collection, control and delivery can enhance the job market and impact on the transportation system. Furthermore, the installation and operation of $A D$ plants would generate new jobs and increase taxation revenue.

One of the main challenges in this study was the limited data availability, mainly due to commercial issues, and hence the simulated effects within the WEF nexus were restricted to the available data relevant to $A D$ operation. 
Whilst estimates of potential costs avoided were mentioned in Section 4.2, the economic impacts in the ABM (Equation 3) excluded incomes from sale revenues (e.g. supply of local energy and digestate) and costs (to the government) of potential financial subsidies which also raise unaccounted impacts. The first exclusion is due to the lack of reliable data as income is usually considered confidential by $A D$ operators and is not shared publicly. The second exclusion is because environmental policies affecting the AD industry can change abruptly depending on voting behaviour, government politics and resulting public policy. These changes cannot be easily predicted or tied to a particular scenario.

Positive social impacts may arise from avoided generation of energy from fossil fuels. Overall public health is influenced by the quality of local air. Previous research found that life expectancies can increase as a result of carbon emission reduction, due to the decrease of PM (Particulate Matter) to 2.5 concentration (Krewski, 2009). The PM, which consists of solid and liquid particles and droplets in the air caused by various sources including the combustion of fossil fuels, was not quantified by the ABM.

\subsection{Future work}

In addition to accounting for some of the invisible impacts such as job creation, and sales, which could be addressed if appropriate data or proxies were made available, there are other improvements that can be considered.

The ABM design can be improved to account for the variation in people's perception, across the years, in regard to $A D$ implementation. This should increase the acceptability of the technology and result in greater diffusion and improving the social impact, particularly in Share $\&$ Connect.

The creation of sub-models within the ABM environment will reflect the spatial variation in resilient approaches and quantify the implications of this for resilience and sustainability across the WEF nexus. This will enable the simulation of scenarios where responses to climate change may differ spatially, e.g. in Create \& Cope. The ABM can be made more granular. Current computer processing speeds do not permit a short enough run time to achieve greater granularity.

The ABM can be used for other future scenarios, for example, those described in the National Grid (2018) that reflect the degrees of energy tensions caused by decarbonisation and digitalisation.

Technology capability has remained static to 2050. It is expected, that the efficiency of AD will increase over time in line with other technological developments.

Other food waste sources, such as social infrastructures, farm waste, food production and wastewater activated sludge can be considered. The ABM employed AD plants which responded to the available feedstock, so new feedstock sources would increase outputs. 


\section{Conclusions}

This study has identified legislation on climate change, which is driving decarbonisation, and has suggested that $A D$ is a suitable technology as part of a hybrid solution which will have positive impacts on the water-energy-food nexus and on social, economic and environmental outcomes. The research considered multiple relationships; intersectoral dependencies and interactions; different scales of change; and an attempt to embrace the complexity of potential changes in three major resource domains.

An $A B M$ was developed to understand $A D$ deployment on a $G B$ scale and reflect the complexity of diffusion upon social, economic and environmental outcomes. This required gathering a huge array of data, from various stakeholders which had varying quality; different levels of granularity; different time series and associated access challenges. Insights from running the $A B M$ under two scenarios were compared to baseline results. The use of the scenarios in the $A B M$ required careful translation of information from the scenario narratives to provide robust and plausible ABM parameters. It required deep discussions across a distinct disciplinary divide (soft climate change futures and hard technology simulation) to build bridges between interpretivist and positivist research methods. These different, and typically opposing approaches, were partially integrated, providing a novel and significant contribution to the literature on $A D$ innovation with relevance to $G B$, and other countries and regions where $A D$ features in the renewable energy plans.

The results demonstrate the range in variability of benefits created by future scenarios. Environmental, economic and social aspects were assessed as well as sectoral impacts on water, energy and food, thus the ABM can be utilised as a tool to aid decision making.

\section{Acknowledgments}

The authors thank the Engineering and Physical Sciences Research Council (EPSRC) for funding the Stepping UP project (EP/N00583X/1). The authors also thank the Stepping UP team, particularly Dr Claire Hoolohan and Professor Alice Larkin (both from the University of Manchester, UK) for their support on interpreting the scenarios and Dr James Suckling (University of Surrey, UK) for his support on case studies which helped identify rules for the ABM.

\section{References}

Albrecht, T., Crootof, A. \& Scott, C. (2018). The Water-Energy-Food Nexus: A systematic review of methods for nexus assessment. Environmental Research Letters, 13(4), 043002, DOI: 10.1088/1748-9326/aaa9c6.

Banks, C., Chesshire, M., Heaven, S., \& Arnold, R. (2011). Anaerobic digestion of sourcesegregated domestic food waste: Performance assessment by mass and energy balance. Bioresource Technology, vol. 102(2), pp 612-20. DOI:10.1016/j.biortech.2010.08.005.

Batstone, D., \& Virdis, B. (2014). The role of anaerobic digestion in the emerging energy economy. Current Opinion in Biotechnology, vol. 27, pp 142-149. DOI: 10.1016/j.copbio.2014.01.013.

Bazilian M., Rogner, H., Howells, M., Hermann, S., Arent, D., Gielen, D., Steduto, P., Mueller, A., Komor, P., Tol, R.S.J., \& Yumkella, K.K. (2011). Considering the energy, water and food nexus: Towards an integrated modelling approach. Energy Policy, vol. 39(12) pp 7896-7906. DOI: 10.1016/j.enpol.2011.09.039. 
BEIS (Department for Business, Energy \& Industrial Strategy) (2018a). Energy Consumption in the UK (ECUK) 2018 Data Tables. Available in: https://www.gov.uk/government/statistics/energy-consumption-in-the-uk.

BEIS (Department for Business, Energy \& Industrial Strategy) (2018b). Greenhouse gas reporting: conversion factors $2018 . \quad$ Available in https://www.gov.uk/government/publications/greenhouse-gas-reporting-conversionfactors-2018.

Cardellini, V., Casalicchio, E., \& Galli, E. (2007). Agent-based modeling of interdependencies in critical infrastructures through UML. Proceedings of the 2007 spring simulation multiconference.

Casalicchio, E., Galli, E., \& Tucci, S. (2007). Federated agent-based modeling and simulation approach to study interdependencies in IT critical infrastructures. 2007 11th IEEE International Symposium on Distributed Simulation and Real-Time Applications (DSRT'07), pp. 182-189.

Cohen, D. (2016). Revealed, the full scale of supermarket waste. Evening Standards- Eating Out Statistics. Available in https://www.standard.co.uk/news/foodforlondon/revealed-thefull-scale-of-supermarket-waste-a3348381.html [Accessed 20 August 2018].

Evangelisti, S., Lettieri, P., Borello, D., \& Clift, R. (2014). Life cycle assessment of energy from waste via anaerobic digestion: A UK case study. Waste Management, vol. 34, pp226237. DOI: 10.1016/j.wasman.2013.09.013

Eyre, N., \& Baruah, P. (2015). Uncertainties in future energy demand in UK residential heating. Energy Policy, vol. 87, pp 641-653. DOI: 10.1016/j.enpol.2014.12.030.

Faber, A., Valente, M., Janssen, P. (2010). Exploring domestic micro-cogeneration in the Netherlands: an agent-based demand model for technology diffusion. Energy Policy, vol. 38(6), pp 2763-2775. DOI: 10.1016/j.enpol.2010.01.008.

Fernández-González, J. M., Grindlay, A. L., Serrano-Bernardo, F., Rodríguez-Rojas, M. I., \& Zamorano, M. (2017). Economic and environmental review of Waste-to-Energy systems for municipal solid waste management in medium and small municipalities. Waste Management, vol. 67, pp 360-374. DOI: 10.1016/j.wasman.2017.05.003.

Green, J.M.H., Cranston, G.R., Sutherland, W.J., Tranter, H.R. Bell, Sarah J. Benton, T.G., Blixt, E., Bowe, C., Broadley, S., Brown, A., Brown, C., Burns, N., Butler, D., Collins, H. Crowley, H., DeKoszmovszky, J., Firbank, L.G., Fulford, B., Gardner, T.A., Hails, R.S., Halvorson, S., Jack. M., Kerrison, B., Koh, L.S.C., Lang, S.C., McKenzie, E.J., Monsivais, P., O'Riordan, T., Osborn, J., Oswald, S., Price Thomas, E., Raffaelli, D., Reyers, B., Srai, J.S., Strassburg, B.B.N., Webster, D., Welters, R., Whiteman, G., Wilsdon, J., \& Vira, B. (2017). Research priorities for managing the impacts and dependencies of business upon food, energy, water and the environment. Sustainability Science, vol. 12(2), pp.319-331. DOI: 10.1007/s11625-016-0402-4.

Grigoryev, I. V. (2015). AnyLogic 7 in Three Days, 2nd ed. CreateSpace Independent Publishing Platform.

Grimm, V., Berger, U., Bastiansen, F., Eliassen S., Ginot, V., Giske, J., Goss-Custard, J., Grand, T., Simone K., Huse, G., Huth, A., Jepsen, J., Jørgensen, C., Mooijh, W., Müllera, B., Pe'eri, G., Piou C., Railsback, S., Robbins, A., Rossmanith, E., Rüger, N., Strand, E., Souissi, S., Stillman, R., Vabø, R., Visser, U., \& DeAngelisn, D. (2006). A standard protocol for describing individual-based and agent-based models. Ecological Modelling, vol. 198(1-2), pp 115-126. DOI: 10.1016/j.ecolmodel.2006.04.023.

Grimm, V., Berger, U., DeAngelis, D.L., Polhill, J.G., Giske, J., \& Railsback, S.F. (2010). The ODD protocol: A review and first update. Ecological Modelling, vol. 221(23), pp 27602768. DOI: 10.1016/j.ecolmodel.2010.08.019.

Haltas, I., Suckling, J., Soutar, I., Druckman, A., \& Varga, L. (2017). Anaerobic digestion: a prime solution for water, energy and food nexus challenges. Energy Procedia, vol. 123, pp 22-29. DOI: 10.1016/j.egypro.2017.07.280

Hatfield-Dodds, S., Schandl, H., Adams, P.D., Baynes, T.M., Brinsmead, T.S., Bryan, B.A., 
Chiew, F.H.S., Graham, P.W., Grundy, M., Harwood, T., McCallum, R., McCrea, R., McKellar, L.E., Newth, D., Nolan, M., Prosser, I., \& Wonhas, A. (2015). Australia is 'free to choose' economic growth and falling environmental pressures. Nature, vol. 527, pp 49-53, DOI: 10.1038/nature16065.

Hoolohan, C., Soutar, I., Suckling, J., Druckman, A., Larkin, A., \& McLachlan, C. (2018). Stepping-up innovations in the water-energy-food nexus: A case study of anaerobic digestion in the UK. The geographical Journal, vol. 185 (2), pp 1-15. DOI: 10.1111/geoj.12259.

Hoolohan, C., McLachlan, C., \& Larkin, A. (2019). 'Aha' moments in the water-energy-food nexus: A new morphological scenario method to accelerate sustainable transformation. Technological Forecasting \& Social Change, vol. 148, 119712. DOI: 10.1016/j.techfore.2019.119712.

Iturriza, M., Labaka, L., Sarriegi, J. M. \& Hernantes, J. (2018). Modelling methodologies for analysing critical infrastructures. Journal of Simulation, vol. 12 (2), pp. 1-16. DOI: 10.1080/17477778.2017.1418640.

Kaddoura, S., \& El Khatib, S. (2017). Review of water-energy-food Nexus tools to improve the Nexus modelling approach for integrated policy making. Science of The Total Environment, vol. 574, pp 1131-1139. DOI: 10.1016/j.scitotenv.2016.09.046.

Kaegi, M., Mock, R., \& Kröger, W. (2009). Analyzing maintenance strategies by agent-based simulations: A feasibility study. Reliability Engineering and System Safety, vol. 94 (9), pp 1416-1421. DOI: 10.1016/j.ress.2009.02.002.

KPI (Key Performance Indicators) Library (2017). Truck Loading Percentages. Available in http://kpilibrary.com/kpis/of-truckload-capacity-utilized [Accessed 18 August 2018].

Krewski, D. (2009). Evaluating the effects of ambient air pollution on life expectancy. The New England Journal of Medicine, vol. 360, pp 413-415. DOI: 10.1056/NEJMe0809178.

Lauer, M., Hansen, J., Lamers, P., \& Thrän, D. (2018). Making money from waste: The economic viability of producing biogas and biomethane in the Idaho dairy industry. Applied Energy, vol. 222, pp 621-636. DOI:10.1016/j.apenergy.2018.04.026.

Levinson, D., Corbett, M., \& Hashami, M. (2004). Operating Costs for Trucks. Retrieved from the University of Minnesota Digital Conservancy, http://hdl.handle.net/11299/179830.

Li, F., \& Trutnevyte, E. (2017). Investment appraisal of cost-optimal and near-optimal pathways for the UK electricity sector transition to 2050. Applied Energy, vol. 189, pp 89109. DOI: 10.1016/j.apenergy.2016.12.047.

Momeni, A., Prasad, V., Dharmawardena, H., Piratla, K., \& Venayagamoorthy, K. (2018). Mapping and Modeling Interdependent Power, Water, and Gas Infrastructures. 2018 Clemson University Power Systems Conference (PSC). DOI:10.1109/PSC.2018.8664050.

National Grid (2016). The future of gas in the UK. Available in https://futureofgas.uk/

National Grid (2018). Future Energy Scenarios: System Operator. Available in http://fes.nationalgrid.com/

Ouyang (2014). Review on modeling and simulation of interdependent critical infrastructure systems. Reliability Engineering and System Safety, vol. 121, pp 43-60. DOI:10.1016/j. ress.2013.06.040.

Pacetti, T., Lombardi, L., \& Federici, G. (2015). Water-energy Nexus: a case of biogas production from energy crops evaluated by Water Footprint and Life Cycle Assessment (LCA) methods. Journal of Cleaner Production, vol. 101, pp 278-291. DOI: 10.1016/j.jclepro.2015.03.084.

Rinaldi, S. (2004). Modeling and simulating critical infrastructures and their interdependencies. 2004 37th Annual Hawaii International Conference on System Sciences.

Rigamonti, L., Sterpi, I., \& Grossoa, M. (2016). Integrated municipal waste management systems: An indicator to assess their environmental and economic sustainability. Ecological Indicators, vol. 60, pp 1-7. DOI: 10.1016/j.ecolind.2015.06.022.

Scarlat, N., Fahl, F., Dallemand, J., Monforti, F., \& Motola, V. (2018). A spatial analysis of biogas potential from manure in Europe. Renewable and Sustainable Energy Reviews, vol. 94, pp 915-930. DOI:10.1016/j.rser.2018.06.035. 
Schmied, M., \& Knörr, W. (2012). Calculating GHG emissions for freight forwarding and logistics services. Available in https://www.clecat.org/media/CLECAT Guide on Calculating GHG emissions for fre ight forwarding and logistics services.pdf.

SGC (Swedish Gas-technology Centre) (2012). Basic Data on Biogas. Available in http://www.sgc.se/ckfinder/userfiles/files/BasicDataonBiogas2012.pdf.

Sgroi, F., Di Trapani, A., Foderà, M., Testa, R., \& Tudisca, S. (2015). Economic performance of biogas plants using giant reed silage biomass feedstock. Ecological Engineering, vol. 81, pp 481-487. DOI: 10.1016/j.ecoleng.2015.04.068

Sorda, G., Sunak, Y., \& Madlener, R. (2013). An agent-based spatial simulation to evaluate the promotion of electricity from agricultural biogas plants in Germany. Ecological Economics, vol. 89(C), pp 43-60. DOI: 10.1016/j.ecolecon.2013.01.022.

Tan, S., Ho, W., Hashim, H., Lee, C, Taib, R., \& Ho, S. (2015). Energy, economic and environmental (3E) analysis of waste-to-energy (WTE) strategies for municipal solid waste (MSW) management in Malaysia. Energy Conversion and Management, vol. 102, pp 111-120. DOI: 10.1016/j.enconman.2015.02.010.

The Caterer (2005). Hospitality industry. Available in: https://www.thecaterer.com/articles/40685/hospitality-industry-faq [Accessed 22 September 2018].

The Scottish Government (2017). Energy in Scotland 2017. Available in http://www.gov.scot/Resource/0051/00514474.pdf.

The UK Government (2011). The Carbon Plan: Delivering our low carbon future. Available in https://www.ukgbc.org/sites/default/files/3702-the-carbon-plan-delivering-our-lowcarbon-future.pdf.

Thomas, E. (2017). ADBA publish pipeline data. Available in http://adbioresources.org/news/tag/758-pipeline-data [Accessed 20 February 2018].

Tom, Q., \& Andrew, P. (2017). Household food waste in the UK, 2015. Available in http://www.wrap.org.uk/sites/files/wrap/Household food waste in the UK 2015 Repo rt.pdf.

Toth, A., Rendall, S., \& Reitsma, F. (2016). Resilient food systems: a qualitative tool for measuring food resilience. Urban Ecosystems, vol. 19(1), pp 19-43. DOI: 10.1007/s11252-015-0489-x.

Tran, M. (2012). Agent-behaviour and network influence on energy innovation diffusion. Communications in Nonlinear Sciences and Numerical Simulations, vol. 17(9), pp 36823695. DOI: 10.1016/j.cnsns.2012.01.016

UN-Population (United Nations Population Division) (2018). World Population Prospects 2017. Available in: https://population.un.org/wpp/Graphs/Probabilistic/POP/TOT/.

Walker, M., Theaker, H., Yaman, R., Poggio, D., Nimmo, W., Bywater, A., \& Pourkahanian, M. (2017). Assessment of micro-scale anaerobic digestion for management of urban organic waste: A case study in London, UK. Waste Management, vol. 61, pp 258-268. DOI: 10.1016/j.wasman.2017.01.036.

Whiting, A., \& Azapagic, A. (2014). Life cycle environmental impacts of generating electricity and heat from biogas produced by anaerobic digestion. Energy, vol. 70, pp 181-193. DOI: 10.1016/j.energy.2014.03.103.

WRAP (Waste \& Resources Action Programme) (2012). Using quality anaerobic digestate to benefit crops. Available in http://www.wrap.org.uk/sites/files/wrap/Quality.

Zio, E., \& Sansavini, G. (2011). Modeling interdependent network systems for identifying cascade-safe operating margins. IEEE Transactions on Reliability, vol. 60 (1), pp 94101. DOI: 10.1109/TR.2010.2104211. 


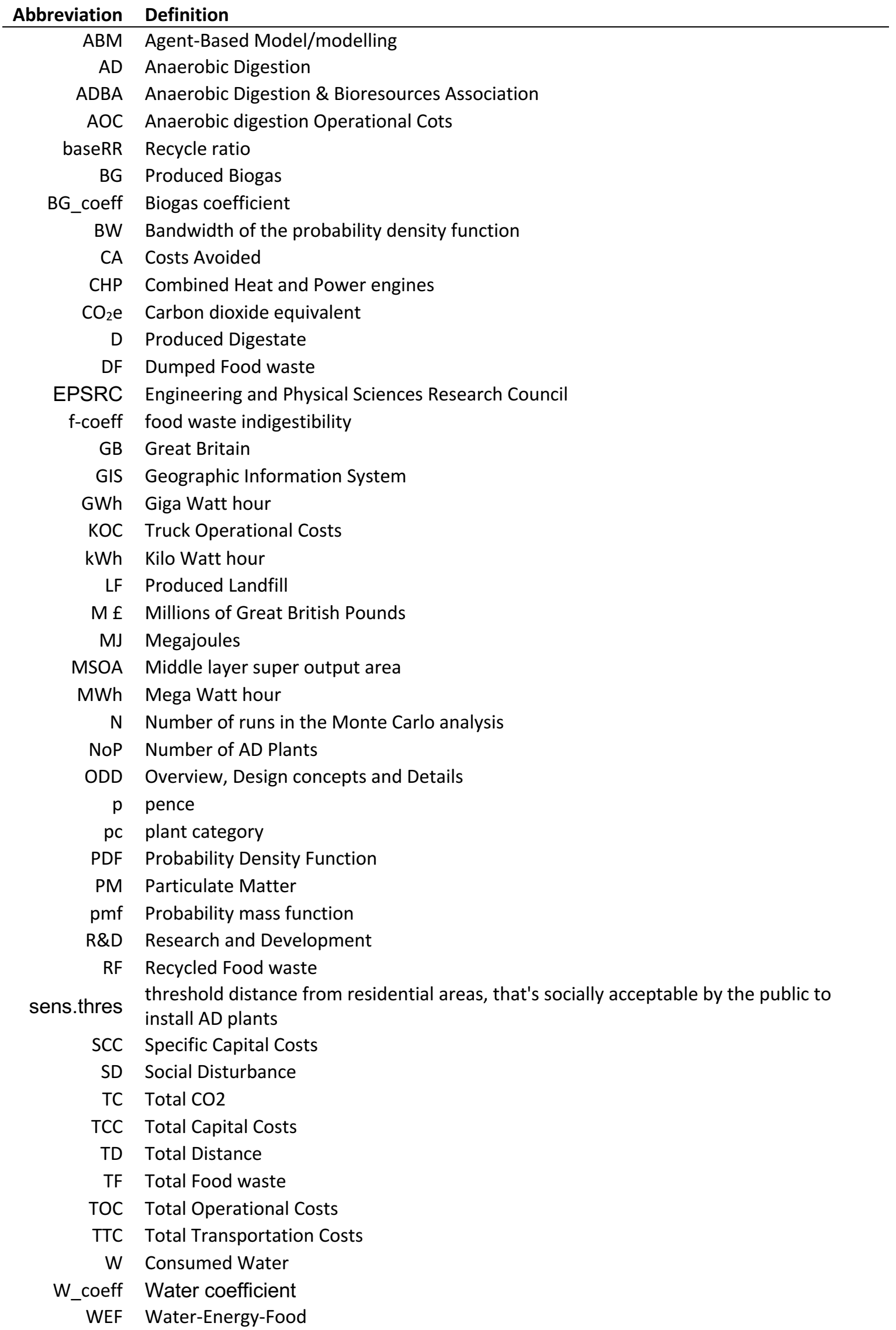

